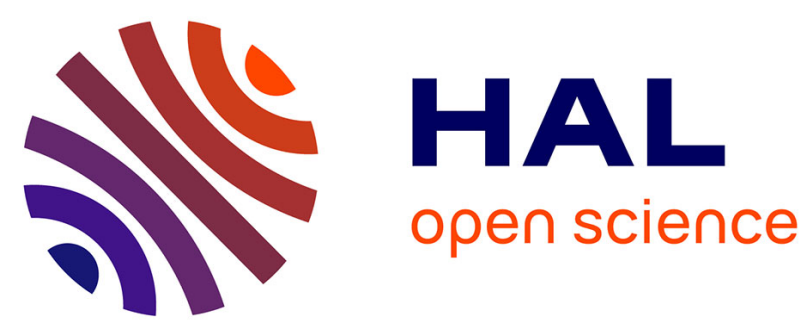

\title{
Analog-Network-Coded Two-Way Relaying Under the Impact of CSI Errors and Network Interference
}

Konstantinos Ntontin, Marco Di Renzo, Christos Verikoukis

\section{To cite this version:}

Konstantinos Ntontin, Marco Di Renzo, Christos Verikoukis. Analog-Network-Coded Two-Way Relaying Under the Impact of CSI Errors and Network Interference. IEEE Transactions on Vehicular Technology, 2016, 65 (11), pp.9029 - 9040. 10.1109/TVT.2016.2517399 . hal-01880143

\section{HAL Id: hal-01880143 https://hal.science/hal-01880143}

Submitted on 7 Jul 2020

HAL is a multi-disciplinary open access archive for the deposit and dissemination of scientific research documents, whether they are published or not. The documents may come from teaching and research institutions in France or abroad, or from public or private research centers.
L'archive ouverte pluridisciplinaire HAL, est destinée au dépôt et à la diffusion de documents scientifiques de niveau recherche, publiés ou non, émanant des établissements d'enseignement et de recherche français ou étrangers, des laboratoires publics ou privés. 


\title{
Analog Network-Coded Two-Way Relaying Under
}

\section{the Impact of CSI Errors and Network Interference}

\author{
Konstantinos Ntontin, Member, IEEE, Marco Di Renzo, Senior Member, IEEE, and \\ Christos Verikoukis, Senior Member, IEEE
}

\begin{abstract}
In this paper, we study a two-way relaying system with Analog Network Coding (ANC) where both the relay and the two sources are under the effect of erroneous Channel State Information (CSI) and network interference originating from a Poisson field of interferers. Our contribution is twofold: 1) We characterize the interference affecting the aforementioned nodes by presenting a general model that incorporates the expected correlation of the interference process affecting the relay with the one affecting the two sources; 2) By deriving the interference statistics, in particular its first moment, we derive analytical formulas for the distribution of the Signal-to-Interferenceplus-Noise Ratio (SINR) of the two sources in the high-SINR region, which reveal that in that region the amount of correlation between the interference processes affecting the relay and the two sources does not affect the SINR distribution. These statistics are subsequently used to derive analytical expressions of the average sum Symbol Error Rate (SER) and achievable average sum rate of the system. Finally, the theoretical results are corroborated by means of Monte Carlo simulations.
\end{abstract}

\section{Index Terms}

Analog Network Coding, Two-Way Relaying Channel, Channel Estimation Errors, Interference, Poisson Point Process.

\section{INTRODUCTION}

Two-way relaying with Analog Network Coding (ANC) has received considerable attention over the recent years due to its ability to reduce by half the amount of time slots needed for the end-to-end communication between two sources, compared to the conventional store-and-forward approach [1]. In

K. Ntontin and C. Verikoukis are with the SMARTECH Department, Telecommunications Technological Centre of Catalonia (CTTC) (e-mail: $\{$ constantinos.ntontin, cveri\}@cttc.es).

M. Di Renzo is with the Laboratory of Signals and Systems (L2S), CNRS-SUPELEC-Univ Paris-Sud, France (e-mail: marco.direnzo@1ss.supelec.fr). 
such a scenario, two sources that want to exchange packets simultaneously send them to an Amplifyand-Forward (AF) relay at the first time slot, which forwards the combined analog signal to the sources at the second time slot. In terms of complexity, using an AF relay is regarded as the simplest scheme compared to other Network Coding approaches [2]-[4] (see also references therein), since it is the only scheme where no further processing is required at the relay other than amplification and forwarding of the incoming signal. Consequently, it is an attractive option for next generation relay-based wireless systems, such as WiMAX and LTE-Advanced in which the operations at the relays should be maintained as low as possible [5].

Up until now, there have been several works on the two-way relaying channel with ANC that deal with different aspects. For instance, regarding coherent systems, as far its information-theoretic capabilities are concerned, in [6] the authors study the optimal beamformers that maximize the achievable rate region in a scenario with multiple relays and they also find the strategies with which the optimal diversity-multiplexing tradeoff is achieved in a scenario with one relay. In addition, in [7] the case of a full-duplex AF relay is studied and it is shown that this case achieves a higher rate than the half-duplex case when the residual self-interference is kept under a certain threshold. Regarding precoding design, [8] studies the joint source and relay precoding design based on the mean-square-error criterion with both the sources and the relay equipped with multiple antennas and in [9] the same authors study a multi-pair of users scenario, which exchange information though a multi-antenna relay with the aim of maximizing the minimum achievable rate among all the users subject to a peak power constraint. For the case of multiple relays that can assist the two-way communication, performance enhancement implementations compared to the single-relay case can be found in the indicative works of [10] and [11] in which relay selection is considered. As far as non-coherent systems are concerned, some indicative works for two-way or one-way relaying are the works of [12]-[15].

All the aforementioned works related to coherent systems consider perfect channel state information (CSI) knowledge at the source nodes, which means that they can perfectly cancel the self-interfering part of the forwarded analog signal from the relay. However, in real world scenarios the CSI needs to be estimated through the use of pilot signals prior to data transmission and, hence, estimation errors are expected, which means that the two sources cannot perfectly eliminate the self-interference of the ANC signal. To this end, the impact of CSI errors on the two-way relaying channel is considered in the work of [16] in which the authors derive analytical frameworks for the outage probability and the bit error rate by 
considering multiple relays, where either maximum ratio combining [17] or relay selection is employed. Moreover, the achievable information rate is derived in [18] by assuming imperfect CSI and, in addition, power allocation based on long-term statistics is proposed, which alleviates its impact. In addition, in [19] relay selection is considered where the relay that is selected is the one that corresponds to the maximum of the worst Signal-to-Noise Ratio (SNR) value between the two source-relay links among all the relays. Analytical error rate expressions are derived for the two sources, which closely match Monte Carlo simulations in the high-SNR region.

Besides imperfect CSI, another performance limitation factor expected to exist in real-world scenarios is co-channel interference. Indicative works of two-way relaying systems considering the impact of Gaussian co-channel interference can be found in [20]-[23]. In particular, in [20] and [21] the outage probability is analytically studied, whereas in [22] besides the outage probability the authors derive analytical expressions for the error probability and the achievable rate. However, these works that consider the impact of cochannel interference assume a perfect CSI knowledge. In contrast, the impact of both CSI errors and co-channel interference is studied in [23] in which the authors derive analytical expressions for the system outage probability, error rate, and achievable sum rate in a two-way multiple relaying system assuming that either the relays dispatch their ANC signal sequentially to the two sources or the relay with the best channel conditions is selected to assist the communication and forward the ANC signal.

Although the consideration of the effect of co-channel interference makes the system analysis more practical, the previously mentioned co-channel interference related works rely on the assumption of Gaussian interference resulting from a finite number of interference. For this to hold, the complex channel coefficients of the interferers should be Gaussian and their position known a priori, which can occur only in planned cellular systems. Consequently, the Gaussian interference assumption seems an implausible case for next generation cellular systems, which are expected to be of heterogeneous nature [24], [25] with several small cells, cognitive radios, and Wi-Fi access points, for instance, overlaid in the area covered by a macro cell. These nodes can act as interferers to the macro-cell users and their number and position are unknown a priori. Hence, the resulting interference is not expected to be Gaussian distributed. To model it, the most common approach in the literature is the stochastic geometry approach in which the location of the interferers and their number are distributed according to a Poisson Point Process (PPP) [26]-[28] .

In light of an increasing amount of works investigating the impact of the PPP interference modeling

\footnotetext{
${ }^{1}$ Although it is not the most realistic stochastic geometry model to describe the expected interference in emerging heterogeneous networks, PPP modeling is preferred due to its analytical tractability and the important insights that the system designer can obtain by its analysis [29].
} 
in future wireless networks, some papers have recently started investigating the performance of one way relay-based systems in the presence of this interference type [30]-[35]. In [30], the outage probability of optimal, maximum ratio, and selection combining [17] is investigated. In [31], the outage probability and error rate of $\mathrm{AF}$-based dual-hop relaying are studied without the inclusion of the direct link from the source to the destination. In [32], the achievable spatial-contention diversity order of cooperative relaying is computed. In [33], the error probability and diversity order of multi-hop relaying are studied assuming that the interference processes affecting the nodes are independent. In [34], the analysis of [31] is extended by considering also the direct link from the source to the destination where maximum ratio combining is used to combine the direct and the relay-forwarded signal. Finally, in [35] the authors consider the same system model as the one in [34], but the selection combining scheme is used at the destination. We note that in [31], [34], and [35] it is assumed that the interference affects only the destination, but not the relay, which would be a more realistic approach.

As aforementioned, these works consider one-way relay systems and to the best of our knowledge there is no work in the literature considering the impact of co-channel interference in the two-way relaying scenario that is characterized by a PPP.

Contribution: This work is motivated by the importance of the two-way relaying scenario, and, hence, the need of the system designer to be in possession of accurate analytical frameworks that lead to insights for importance performance metrics, such as the average error rate and achievable rate. By jointly taking into account for the first time in this scenario practical aspects, such as imperfect channel estimates and interfering nodes randomly distributed whose number and location are not known a priori, we provide the following two-fold contribution:

1) Against the simplified assumptions of previous works on the two-way relaying channel with ANC that they either consider perfect channel estimates or Gaussian-distributed interference statistics, which largely deviate from the expected statistics of randomly deployed interfering nodes, in this work we take both of these practical aspects into account. Regarding the interference, we consider PPP-based co-channel interference affecting both the relay and the two sources, which incorporates the expected correlation between the processes affecting these nodes. This correlation arises due to same interferers expected to be active at both time slots that the communications lasts without changing their position.

2) High-SINR formulas are derived for the average sum SER and achievable average sum rate of the two sources by first computing their SINR distribution in the specific region. This distribution involves 
the first moment of the interference processes that affect the relay and the two sources and it is not dependent on the amount of correlation between them. These derived analytical formulas are validated by means of Monte Carlo simulations, which exhibit a relatively close match with the analysis in the high-SINR region. In addition, important insights are obtained based on the analytical expressions, which are numerically validated in Section IV.

Organization: The remainder of this paper is organized as follows. In Section II, the system model under study is presented and the network interference is characterized. Section III presents the performance analysis that leads to closed-form expressions of the average sum SER and achievable average sum rate of the two sources. Section IV validates the analytical results by comparing them with their Monte Carlobased counterparts and observing their relatively close match in the high-SINR region. Finally, Section V concludes this work and presents guidelines for future work on this area.

Notation: The following notation is used throughout this paper. i) $E_{x}\{\cdot\}$ denotes the mean value of the stochastic process $x$; ii) $|\cdot|$ is the absolute value of a complex number; iii) (.) denotes the binomial coefficient; iv) $B(\cdot, \cdot)$ is the beta function; v) $\ln (\cdot)$ denotes the natural logarithm; vi) $C N\left(\mu, \sigma^{2}\right)$ denotes a complex Gaussian random variable with mean equal to $\mu$ and variance equal to $\sigma^{2}$; vii) ${ }_{2} F(\cdot, \cdot, \cdot, \cdot)$ is the Gaussian hypergeometric function; viii) $(r)_{k}=r(r-1) \cdots(r-k+1)$ denotes the falling factorial; ix) $\operatorname{Pr}(\cdot)$ denotes probability, and $\mathrm{x}) K_{1}(\cdot)$ is the modified Bessel function of the second kind and first order.

\section{System Model}

In this section, we present the scenario under study and its signal model, characterize the interference that affects the relay and the sources, and derive approximate expressions of the instantaneous SINR of the two sources.

\section{A. Scenario}

We assume that two fixed single-antenna sources, which we denote as $A$ and $B$, want to exchange packets in a narrowband flat-fading environment via a fixed half-duplex, single-antenna, AF, and variablegain relay. Furthermore, we assume that the channel coefficients do not change during the channel estimation and data communication phase. For a better utilization of the network resources, we allow the two packets to be analog network-coded and, hence, the end-to-end communication occurs in two phases: i) During the first phase, which has a duration of one time slot, the two sources simultaneously 
dispatch their packets to the relay. ii) During the second phase, which also has a duration of one time slot, the relay amplifies the summed signal received from the two sources and dispatches it to them. Subsequently, each source detects its intended packet after subtracting its self-interfering signal from the received signal. For this, we assume that each source has an imperfect estimate of its corresponding channel coefficient to the the relay. In addition, we also assume that the relay has imperfect channel estimates of the channel coefficients from the sources to itself.

Moreover, we assume that both the relay and the sources are affected by PPP homogeneous interference ${ }^{2}$ with intensity $\lambda\left(\frac{\text { nodes }}{m^{2}}\right)$ at both time slots that the communication lasts and for simplification, without loss of generality, that the relay is located at the origin of the network and sources $A$ and $B$ are at a distance $d_{A}$ and $d_{B}$ from the relay, respectively. In addition, we consider that transmissions from interferers (nodes that use the same frequency with the two-way system under consideration) located inside a circle with radius $R_{\text {exc }}>\max \left(d_{A}, d_{B}\right)$ measured from the origin are inhibited. This assumption is made to eliminate the possibility of having co-channel interferers arbitrarily close to any of the source nodes, which would result in very strong interference (infinite in theory), and can be practically feasible through proper sensing and resource allocation. Hence, we assume that there is an exclusion region regarding the interference. This system model is depicted in Fig. 1, where the triangles represent the possible sources of the homogeneous interference.

We further assume that the interferers have the same transmit power level, which we denote as $P_{I}$. These interferers affect the relay at the first time slot and at the second slot the two sources. Furthermore, we denote the transmission power level of source $A$ as $P_{A}$ and the one of source $B$ as $P_{B}$. Finally, we consider an unbounded path-loss model where the received power at a node from another node that is located at a distance $d$ scales with $1 / d^{a}$, where $a$ is the path-loss exponent ${ }^{3}$.

\section{B. Signal Model}

Let us now present the signal model of two communication phases of the system.

\footnotetext{
${ }^{2}$ In reality, the interferers can be of various types, such as macro and small-cell base stations, mobile terminals, and the AF relays, which makes the interference heterogeneous. However, we make the assumption that the power level of the interfering base stations is much stronger than the power level of the rest types of interferers (due also to the assumption of an exclusion region for the interference) and, hence, the only interfering effect that can be considered is the one of the base stations. Consequently, the PPP interference becomes homogeneous by assuming for simplicity that the power level of all the interfering nodes is the same.

${ }^{3}$ Although the unbounded path-loss model is unrealistic for very small distances between nodes, if a minimum distance between the interferers and the relay and source nodes is maintained throughout the communication period (which can be the case due to the exclusion region), both the unbounded and the bounded path loss models would tend to give the same values of the performance metrics [36].
} 


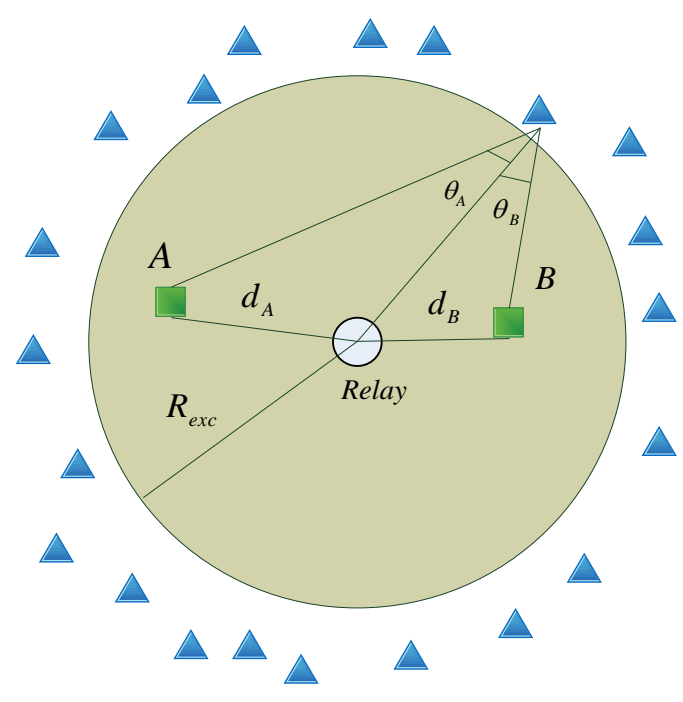

Fig. 1: System Model.

First phase: The signal received at the relay in the first time slot, which we denote as $y_{R}$, is given by

$$
y_{R}=\sqrt{\frac{P_{A}}{d_{A}^{a}}} h_{A} s_{A}+\sqrt{\frac{P_{B}}{d_{B}^{a}}} h_{B} s_{B}+n_{R}+i_{R},
$$

where $s_{A}$ and $s_{B}$ are the modulated packets of source $A$ and source $B$, respectively, $h_{k} \sim C N\left(0, \sigma_{h_{k}}^{2}\right)$ (Rayleigh fading assumption) denotes the channel coefficient of source $k$, where $k=\{A, B\}$ (notation that is used as throughout this paper) $n_{R} \sim C N(0,1)$ is the Additive White Gaussian Noise (AWGN) at the relay, and $i_{R}$ represents the interfering signal at the relay. In addition, we assume that

$$
h_{k}=\hat{h}_{k}+e_{h_{k}}
$$

where $\hat{h}_{k}$ denotes the estimated channel coefficient of source $k$ of which the relay and the sources are aware and $e_{h_{k}} \sim C N\left(0, \sigma_{e_{h_{k}}}^{2}\right)$ denotes the Gaussian distributed channel estimation error of the source $k$-relay link, which we assume to be independent of $\hat{h}_{k}{ }^{4}$. Hence, $\hat{h}_{k} \sim C N\left(0, \sigma_{h_{k}}^{2}-\sigma_{e_{h_{k}}}^{2}\right)$. One question that arises is how it is possible that both the relay and the sources have exactly the same erroneous channel estimates. For this to occur, we assume that the relay is the one that performs channel estimation, obtains the erroneous $\hat{h}_{k}$ channel estimates of the two sources, and subsequently forwards them to the sources prior to data transmission.

Second phase: In the second phase, the variable-gain relay first amplifies $y_{R}$ after proper power scaling.

${ }^{4}$ This can be the case when $\hat{h}_{k}$ is the minimum mean square error estimate of $h_{k}$ [16]. 
By assuming that the relay is unaware of the interference statistics (including the long-term statistics), but aware of $\sigma_{e_{h_{k}}}^{2}$, it employs the amplification factor that makes the instantaneous transmitted power from the relay equal to $P_{R}$ in the absence of interference. We denote it by $G_{R}$ and it is given by [16, Eq. (3)]

$$
G_{R}=\sqrt{\frac{P_{R}}{\frac{P_{A}}{d_{A}^{a}}\left(\left|\hat{h}_{A}\right|^{2}+\sigma_{e_{h_{A}}}^{2}\right)+\frac{P_{B}}{d_{B}^{a}}\left(\left|\hat{h}_{B}\right|^{2}+\sigma_{e_{h_{B}}}^{2}\right)}} .
$$

For simplicity, we have excluded the scaling of $G_{R}$ with the unit variance of the noise at the relay due to the fact that its effect is negligible for high $P_{A} / d_{A}^{a}$ and $P_{B} / d_{B}^{a}$ values (high-SNR region). Subsequently, after amplification, the relay dispatches the received signal to the sources.

The received signal of source $k$, which we denote as $y_{k}$, is given by

$$
y_{k}=G_{R} y_{R} \frac{h_{k}}{\sqrt{d_{k}^{a}}}+n_{k}+i_{k},
$$

where $n_{k} \sim C N(0,1)$ and $i_{k}$ denote the AWGN and interference, respectively, that affect source $k$ at the second time slot. By having the channel estimate $\hat{h}_{k}$, source $k$ performs imperfect self-interference cancellation and, hence, the resulting signal, which we denote as $y_{k}^{\prime}$, is given by

$$
y_{k}^{\prime}=y_{k}-G_{R} \frac{\sqrt{P_{k}}}{d_{k}^{a}} \hat{h}_{k}^{2} s_{k}
$$

By substituting (4) into (5), we get

$$
\begin{aligned}
y^{\prime} & =2 G_{R} \frac{\sqrt{P_{k}}}{d_{k}^{a}} \hat{h}_{k} e_{h_{k}} s_{k}+G_{R} \frac{\sqrt{P_{k}}}{d_{k}^{a}} e_{h_{k}}^{2} s_{\bar{k}}+G_{R} \sqrt{\frac{P_{\bar{k}}}{d_{k}^{a} d_{\bar{k}}^{a}}} \hat{h}_{k} \hat{h}_{\bar{k}} s_{\bar{k}}+G_{R} \sqrt{\frac{P_{\bar{k}}}{d_{k}^{a} d_{\bar{k}}^{a}}} \hat{h}_{\bar{k}} e_{h_{k}} s_{\bar{k}} \\
& +G_{R} \sqrt{\frac{P_{\bar{k}}}{d_{k}^{a} d \bar{a}}} \hat{h}_{k} e_{h_{\bar{k}}} s_{\bar{k}}+G_{R} \sqrt{\frac{P_{\bar{k}}}{d_{k}^{a} d_{\bar{k}}^{a}}} e_{h_{k}} e_{h_{\bar{k}}}+G_{R} \frac{n_{R} \hat{h}_{k}}{\sqrt{d_{k}^{a}}}+G_{R} \frac{n_{R} e_{h_{k}}}{\sqrt{d_{k}^{a}}} \\
& +G_{R} \frac{i_{R} \hat{h}_{k}}{\sqrt{d_{k}^{a}}}+G_{R} \frac{i_{R} e_{h_{k}}}{\sqrt{d_{k}^{a}}}+n_{k}+i_{k},
\end{aligned}
$$

where $\bar{k}=B, A$ if $k=A, B$, respectively. According to (6), the useful signal is $G_{R} \sqrt{\frac{P_{\bar{k}}}{d_{k}^{a} d \frac{h}{h}}} \hat{h}_{k} \hat{h}_{\bar{k}} s_{\bar{k}}$ and the rest of terms constitute the interference plus the noise.

\section{Interference Characterization}

Now, let us discuss about the interference processes $i_{R}$ and $i_{k}$ that affect the relay and source $k$, respectively. The most general assumption is that $i_{R}$ is correlated with $i_{k}$ since in real-world scenarios it is expected that some interferers that are active at the first time slot are also active at the second time slot 
and their location remains the same in these two slots. In addition, some interferers that are active at the first time slot are not at the second one and, in the same way, there are new interferers generated at the second time slot that are not active at the first one. This scenario can be described by two probabilities, $m_{1}, m_{2} \leq 1$, where $m_{1}$ is the probability that the interferers are active at both time slots and $m_{2}=1-m_{1}$ is the probability that the interferers are only active either at the first time slot or at the second one. Based on this and by considering the superposition and thinning property of a PPP [37], $i_{R}$ and $i_{k}$ can be modeled by three pair-wise independent PPPs, $i_{1}, i_{2}$, and $i_{3}$, as

$$
i_{R}=i_{1_{R}}+i_{2}, \quad i_{k}=i_{1_{k}}+i_{3_{k}}
$$

where $i_{1_{R}}$ and $i_{1_{k}}$ correspond to the interferers that are active at both time slots (they belong to the same PPP $i_{1}$ ) and affect the relay and source $k$, respectively. $i_{2}$ corresponds to the interferers that are active only at the first time slot and, hence, they affect only the relay, and $i_{3_{k}}$ to the ones that are active only at the second time slot (they belong to the same PPP $i_{3}$ ) and affect source $k$. Consequently, it holds that $i_{1_{R}}$ and $i_{1_{k}}$ have intensity $\lambda_{1}=m_{1} \lambda$ and the intensity of $i_{2}$ and $i_{3_{k}}$ is $\lambda_{2}=m_{2} \lambda$. Hence, from the above we understand that $i_{R}$ is correlated with $i_{k}$ due to the correlation of $i_{1_{R}}$ with $i_{1_{k}}$. In mathematical terms, we have

$$
\begin{aligned}
& i_{1_{R}}=\sum_{i} \sqrt{\frac{P_{I}}{d_{i}^{a}}} h_{i}, \quad i_{2}=\sum_{l} \sqrt{\frac{P_{I}}{d_{l}^{a}}} h_{l} \\
& i_{1_{k}}=\sum_{i} \sqrt{\frac{P_{I}}{\left(\sqrt{d_{i}^{2}+d_{k}^{2}-2 d_{i} d_{k} \cos \theta_{k_{i}}}\right)^{a}}} h_{k_{i}} \\
& i_{3_{k}}=\sum_{p} \sqrt{\frac{P_{I}}{\left(\sqrt{d_{p}^{2}+d_{k}^{2}-2 d_{p} d_{k} \cos \theta_{k_{p}}}\right)^{a}}} h_{k_{p}},
\end{aligned}
$$

where $d_{i}, d_{l}$, and $d_{p}$ denote the distances between the corresponding interferers and the origin of the network, where we have assumed that the relay is located, and the angles $\theta_{A}$ and $\theta_{B}$ are illustrated in Fig. 1. In addition, we assume that $h_{i}, h_{l}, h_{k_{i}}$, and $h_{k_{p}} \sim C N(0,1)$ (Rayleigh fading) and that they are pair-wise independent.

The instantaneous power effect that $i_{R}$ and $i_{k}$ exhibit on the relay and source $k$, respectively, which we 
denote as $I_{R_{\text {inst }}}$ and $I_{k_{\text {inst }}}$, respectively, is given by

$$
\begin{aligned}
& I_{R_{\text {inst }}}=E\left\{i_{R} i_{R}^{*}\right\}=I_{1_{R_{\text {inst }}}}+I_{2_{\text {inst }}} \\
& I_{k_{\text {inst }}}=E\left\{i_{k} i_{k}^{*}\right\}=I_{1_{k_{\text {inst }}}+}+I_{3_{k_{\text {inst }}}},
\end{aligned}
$$

where

$$
\begin{aligned}
& I_{1_{R_{\text {inst }}}}=\sum_{i} \frac{P_{I}}{d_{i}^{a}}\left|h_{i}\right|^{2}, \quad I_{2_{\text {inst }}}=\sum_{l} \frac{P_{I}}{d_{l}^{a}}\left|h_{l}\right|^{2} \\
& I_{1_{k_{\text {inst }}}}=\sum_{i} \frac{P_{I}}{\left(\sqrt{d_{i}^{2}+d_{k}^{2}-2 d_{i} d_{k} \cos \theta_{k_{i}}}\right)^{a}}\left|h_{k_{i}}\right|^{2} \\
& I_{3_{k_{\text {inst }}}}=\sum_{p} \frac{P_{I}}{\left(\sqrt{d_{p}^{2}+d_{k}^{2}-2 d_{p} d_{k} \cos \theta_{k_{p}}}\right)^{a}}\left|h_{k_{p}}\right|^{2} .
\end{aligned}
$$

The correlation coefficient between the interference processes $I_{R_{\text {inst }}}$ and $I_{k_{\text {inst }}}$, which we denote as $\rho_{R, k}$, is given by

$$
\rho_{R, k}=\frac{E\left\{I_{R_{\text {inst }}} I_{k_{\text {inst }}}\right\}-E\left\{I_{R_{\text {inst }}}\right\} E\left\{I_{k_{\text {inst }}}\right\}}{\sqrt{E\left\{\left(I_{R_{\text {inst }}}\right)^{2}\right\}-\left(E\left\{I_{R_{\text {inst }}}\right\}\right)^{2}} \sqrt{E\left\{\left(I_{k_{\text {inst }}}\right)^{2}\right\}-\left(E\left\{I_{k_{\text {inst }}}\right\}\right)^{2}}} .
$$

When $m_{1}=0$, it holds that $\rho_{R, k}=0$, since $I_{R_{\text {inst }}}$ and $I_{k_{\text {inst }}}$ are independent.

Finally, we note that conditioned on $d_{i}, d_{l}$, and $d_{p}, i_{1_{R}}, i_{2}, i_{1_{k}}$, and $i_{3_{k}}$ are zero-mean Gaussian random variables with respective variances $I_{1_{R}}, I_{2}, I_{1_{k}}$, and $I_{3_{k}}$ given by

$$
\begin{aligned}
& I_{1_{R}}=\sum_{i} \frac{P_{I}}{d_{i}^{a}}, \quad I_{2}=\sum_{l} \frac{P_{I}}{d_{l}^{a}} \\
& I_{1_{k}}=\sum_{i} \frac{P_{I}}{\left(\sqrt{d_{i}^{2}+d_{k}^{2}-2 d_{i} d_{k} \cos \theta_{k_{i}}}\right)^{a}} \\
& I_{3_{k}}=\sum_{p} \frac{P_{I}}{\left(\sqrt{d_{p}^{2}+d_{k}^{2}-2 d_{p} d_{k} \cos \theta_{k_{p}}}\right)^{a}} .
\end{aligned}
$$

\section{Instantaneous SINR}

Now, we are going to derive instantaneous approximate expressions of the SINR of source $k$.

Proposition 1. By considering that in practical cases it holds that $\sigma_{e_{h_{k}}}^{2}, \sigma_{e_{h_{\bar{k}}}}^{2} \ll 1$ and due to the fact that in this work we are interested in average metrics, such as the average sum SER and the achievable 
average sum rate, (6) can be approximated as

$$
\begin{aligned}
y^{\prime} & \approx 2 G_{R} \frac{\sqrt{P_{k}}}{d_{k}^{a}} \hat{h}_{k} e_{h_{k}} s_{k}+G_{R} \sqrt{\frac{P_{\bar{k}}}{d_{k}^{a} d_{\bar{k}}^{a}}} \hat{h}_{k} \hat{h}_{\bar{k}} s_{\bar{k}}+G_{R} \sqrt{\frac{P_{\bar{k}}}{d_{k}^{a} d_{\bar{k}}^{a}}} \hat{h}_{\bar{k}} e_{h_{k}} s_{\bar{k}} \\
& +G_{R} \sqrt{\frac{P_{\bar{k}}}{d_{A}^{a} d_{\bar{k}}^{a}}} \hat{h}_{k} e_{h_{\bar{k}}} s_{\bar{k}}+G_{R} \frac{n_{R} \hat{h}_{k}}{\sqrt{d_{k}^{a}}}+G_{R} \frac{i_{R} \hat{h}_{k}}{\sqrt{d_{k}^{a}}}+G_{R} \frac{i_{R} e_{h_{k}}}{\sqrt{d_{k}^{a}}}+n_{k}+i_{k} .
\end{aligned}
$$

\section{Proof: See APPENDIX A.}

From (13), we observe that conditioned on the distances between the interferers and the relay, between the interferers and source $k$, and on $\hat{h}_{k}$ and $\hat{h}_{\bar{k}}$, the interference plus noise term is Gaussian, since we have assumed that the fast fading channel coefficients from the interferers to the relay and source $k$ are Gaussian. Hence, by considering (3), the SINR of source $k$, which we denote as $\gamma_{k}$, that results from (13) is given by

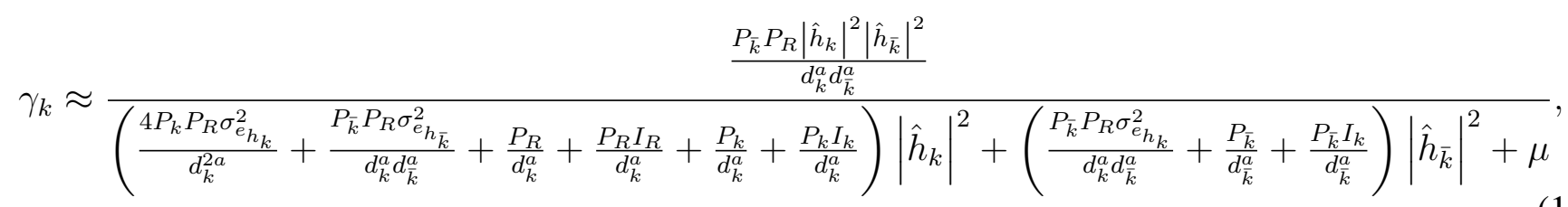

where $I_{R}=I_{1_{R}}+I_{2}, I_{k}=I_{1_{k}}+I_{3_{k}}$, and $\mu=\frac{P_{R} I_{R} \sigma_{h_{k}}^{2}}{d_{k}^{a}}+\frac{P_{k}}{d_{k}^{a}} \sigma_{e_{h_{k}}}^{2}+\frac{P_{\bar{k}}}{d_{\bar{k}}^{a}} \sigma_{e_{\bar{k}}}^{2}+I_{k}\left(\frac{P_{k}}{d_{k}^{a}} \sigma_{e_{h_{k}}}^{2}+\frac{P_{\bar{k}}}{d_{\bar{k}}^{a}} \sigma_{e_{h_{\bar{k}}}}^{2}\right)$.

Proposition 2. By again taking into account that in practical cases it holds that $\sigma_{e_{h_{k}}}^{2}, \sigma_{e_{h_{\bar{k}}}}^{2} \ll 1$, the factor $\mu$ can be excluded from the denominator of (14) since the average value of each of its terms is much smaller than the average value of other terms of the denominator. Hence, (14) can be further approximated (after multiplying the enumerator and denominator with $d_{k}^{a} d_{\bar{k}}^{a}$ ) as

$$
\gamma_{k} \approx \frac{P_{\bar{k}} P_{R}\left|\hat{h}_{k}\right|^{2}\left|\hat{h}_{\bar{k}}\right|^{2}}{C_{k}\left|\hat{h}_{k}\right|^{2}+D_{k}\left|\hat{h}_{\bar{k}}\right|^{2}}
$$

where

$$
C_{k}=4 P_{k} P_{R} \sigma_{e_{h_{k}}}^{2} \frac{d_{\bar{k}}^{a}}{d_{k}^{a}}+P_{\bar{k}} P_{R} \sigma_{e_{h_{\bar{k}}}}^{2}+P_{R} d_{\bar{k}}^{a}+P_{R} I_{R} d_{\bar{k}}^{a}+P_{k} d_{\bar{k}}^{a}+P_{k} I_{k} d_{\bar{k}}^{a}
$$

and

$$
D_{k}=P_{\bar{k}} P_{R} \sigma_{e_{h_{k}}}^{2}+P_{\bar{k}} d_{k}^{a}+P_{\bar{k}} I_{k} d_{k}^{a}
$$

Proof: See APPENDIX B. 


\section{PERFORMANCE ANALYSIS}

In this section, we derive high-SINR expressions of the average sum SER and the achievable average sum rate of source $k$. Towards this, we first derive the Cumulative Density Function (CDF) of the instantaneous SINR in that region and subsequently we use known literature formulas that involve the statistics of the SINR to derive the corresponding performance metrics.

\section{A. SINR Distribution}

Proposition 3. In the high-SINR region, the CDF of (15), which we denote as $F_{k}(x)$, can be approximated as

$$
F_{k}(x)=E_{I}\left\{F_{k}(x \mid I)\right\} \approx N_{k} x
$$

where

$$
N_{k}=\left(\sigma_{h_{\bar{k}}}^{2}-\sigma_{e_{h_{\bar{k}}}}^{2}\right) \frac{d_{\bar{k}}^{a}}{P_{\bar{k}}} E_{I}\left\{I_{R}\right\}+\left[\left(\sigma_{h_{k}}^{2}-\sigma_{e_{h_{k}}}^{2}\right) d_{k}^{a}+\left(\sigma_{h_{\bar{k}}}^{2}-\sigma_{e_{h_{\bar{k}}}}^{2}\right) \frac{P_{k}}{P_{\bar{k}}} d_{\bar{k}}^{a}\right] \frac{E_{I}\left\{I_{k}\right\}}{P_{R}}+Z_{k}
$$

with

$$
\begin{gathered}
Z_{k}=\left(\sigma_{h_{\bar{k}}}^{2}-\sigma_{e_{h_{\bar{k}}}}^{2}\right)\left[4 \frac{P_{k}}{P_{\bar{k}}} \sigma_{e_{h_{k}}}^{2} \frac{d_{\bar{k}}^{a}}{d_{k}^{a}}+\sigma_{e_{h_{\bar{k}}}}^{2}+\left(1+\frac{P_{k}}{P_{R}}\right) \frac{d_{\bar{k}}^{a}}{P_{\bar{k}}}\right]+\left(\sigma_{h_{k}}^{2}-\sigma_{e_{h_{k}}}^{2}\right)\left(\sigma_{e_{h_{k}}}^{2}+\frac{d_{k}^{a}}{P_{R}}\right), \\
E_{I}\left\{I_{R}\right\}=E_{I}\left\{I_{1_{R}}\right\}+E_{I}\left\{I_{2}\right\}=\frac{2 \pi \lambda P_{I}}{a-2} R_{\text {exc }}^{2-a},
\end{gathered}
$$

and

$$
E_{I}\left\{I_{k}\right\}=E_{I}\left\{I_{1_{k}}\right\}+E_{I}\left\{I_{3_{k}}\right\}=A_{I_{k}}
$$


with

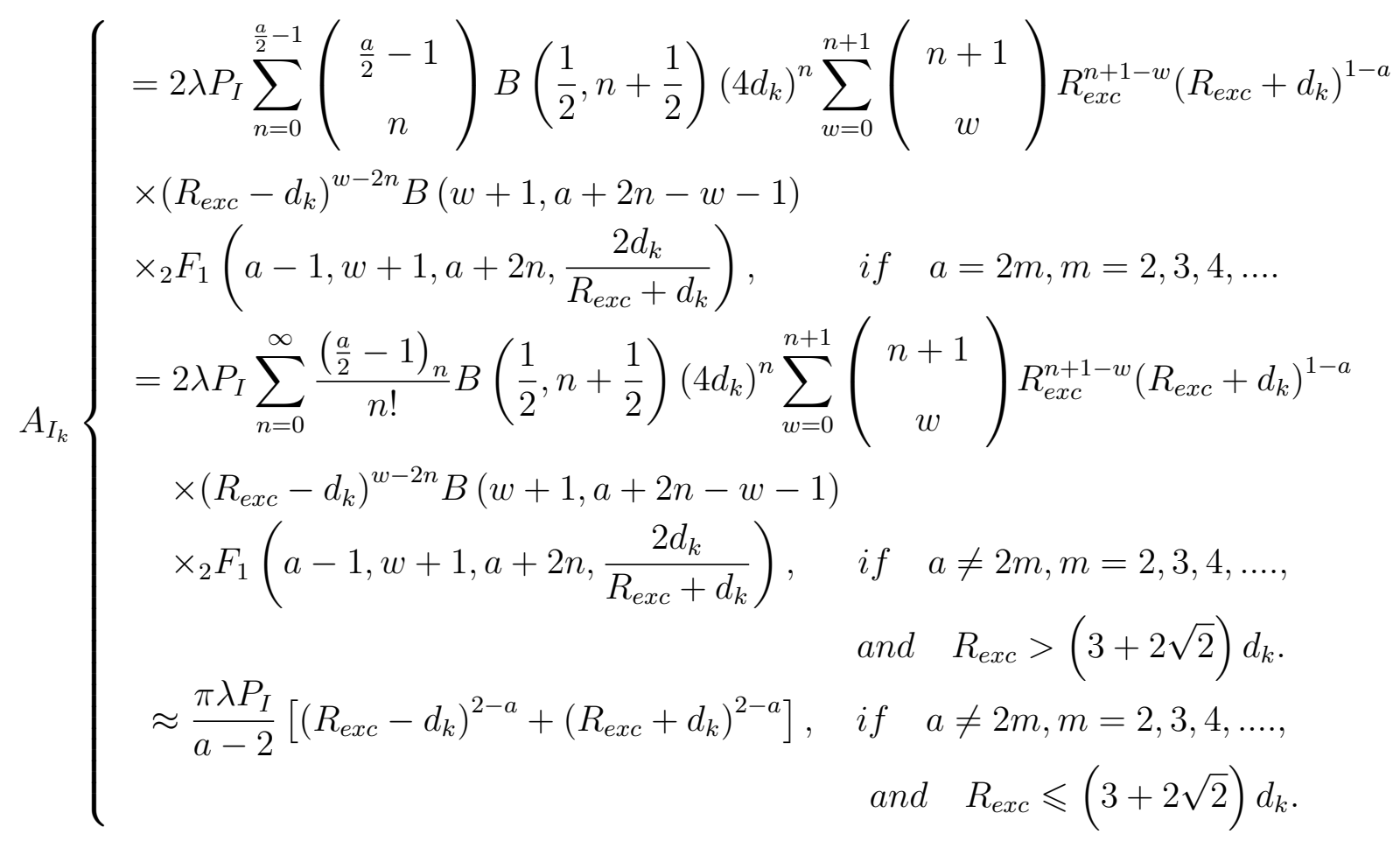

\section{Proof: See APPENDIX C.}

Remark 1. By substituting (21) and (22) into (18), we observe that $F_{k}(x)$ in the high-SINR region does not depend on $m_{1}$ and $m_{2}$. This means that the amount of correlation between the interference processes that affect the relay and source $k$ does not affect $F_{k}(x)$ in that region and, consequently, it does not also affect the performance metrics that are derived based on $F_{k}(x)$, such as the average error rate and achievable rate.

\section{B. Average Sum SER}

The instantaneous SER of a coherent detection system, which we denote as $P_{S_{\text {inst }}}$, in AWGN (or noise+interference that is Gaussian distributed) can be approximated as [17, Eq. (6.73)]

$$
P_{S_{\text {inst }}} \approx \frac{c}{\pi} \int_{0}^{\pi / 2} e^{-\frac{g \gamma_{S I N R}}{\sin ^{2} \phi}} d \phi,
$$

where $\gamma_{S I N R}$ is the instantaneous SINR and $c$ and $g$ are constants that depend on the modulation that is used. For instance, for rectangular Mary-Quadrature Amplitude Modulation (MQAM) it holds that [17, Table 6.1]

$$
c=\frac{4(\sqrt{M}-1)}{\sqrt{M}}, \quad g=\frac{3}{M-1} .
$$


As we aforementioned in Section II-C, conditioned on the distances between the interferers and the relay, between the interferers and source $k$, and on $\hat{h}_{k}$ and $\hat{h}_{\bar{k}}$, the interference plus noise term is Gaussian, since we have assumed that the fast fading complex channel coefficients from the interferers to the relay and source $k$ are Gaussian (Rayleigh fading). Hence, (24) holds in our system model with $\gamma_{S I N R}$ given by (15). From (24), the average SER of source $k$, which we denote as $\bar{P}_{E_{k}}$, can be approximated as [17, Eq. (6.74)]

$$
P_{E_{k}} \approx \frac{c}{\pi} \int_{0}^{\pi / 2} M_{\gamma_{k}}\left(\frac{g}{\sin ^{2} \varphi}\right) d \varphi
$$

where $M_{\gamma_{k}}(s)$ denotes the Moment Generating Function (MGF) of the instantaneous SINR of source $k$, which is given by

$$
M_{\gamma_{k}}(s)=E_{\gamma_{k}}\left\{e^{-s \gamma_{k}}\right\}=\int_{0}^{\infty} e^{-s x} f_{k}(x) d x
$$

where $f_{k}(x)$ is the pdf of the instantaneous SINR of source $k$. In the high-SINR region, we have

$$
f_{k}(x)=\frac{d F_{k}(x)}{d x} \stackrel{(d)}{\approx} N_{k}
$$

where in $(d)$ we use (18).

By substituting (28) into (27), we get

$$
M_{\gamma_{k}}(s) \approx \frac{N_{k}}{s}
$$

Hence, the average sum SER of the two sources, which we denote as $\bar{P}_{S_{\text {sum }}}$, is given by

$$
\begin{aligned}
\bar{P}_{S_{\text {sum }}}=P_{E_{A}}+P_{E_{B}} & \approx \frac{c}{\pi}\left(\int_{0}^{\pi / 2} M_{\gamma_{A}}\left(\frac{g}{\sin ^{2} \varphi}\right) d \varphi+\int_{0}^{\pi / 2} M_{\gamma_{B}}\left(\frac{g}{\sin ^{2} \varphi}\right) d \varphi\right) \\
& \stackrel{(e)}{=} \frac{c}{4 g}\left(N_{A}+N_{B}\right),
\end{aligned}
$$

where in $(e)$ we use (29).

\section{Average Sum Rate}

Since we have proved in Section II-D that conditioned on the distances between the interferers and the relay, between the interferers and source $k$, and on $\hat{h}_{k}$ and $\hat{h}_{\bar{k}}$, the noise plus interference stochastic process at source $k$ (and, consequently, at source $\bar{k}$ due to symmetry) is Gaussian distributed, Shannon's formula [42] holds for the instantaneous maximum achievable rate of sources $A$ and $B$, which we denote 
as $R_{A}$ and $R_{B}$, respectively. Consequently [43, Eq. (30)],

$$
R_{A}=\frac{1}{2} \log _{2}\left(1+\gamma_{A}\right), \quad R_{B}=\frac{1}{2} \log _{2}\left(1+\gamma_{B}\right)
$$

where the $\frac{1}{2}$ multiplier is due to the fact that the duration of the communication is two time slots. Hence, the achievable average sum rate of the system, which we denote as $\bar{R}_{\text {sum }}$, is given by

$$
\begin{aligned}
\bar{R}_{\text {sum }} & =E_{\gamma_{A}}\left\{R_{A}\right\}+E_{\gamma_{B}}\left\{R_{B}\right\}=\frac{1}{2} E_{\gamma_{A}}\left\{\log _{2}\left(1+\gamma_{A}\right)\right\}+\frac{1}{2} E_{\gamma_{B}}\left\{\log _{2}\left(1+\gamma_{B}\right)\right\} \\
& \stackrel{(f)}{=} \frac{1}{2} \log _{2}(e)\left(\int_{0}^{\infty} \frac{1-F_{A}(x)}{1+x} d x+\int_{0}^{\infty} \frac{1-F_{B}(x)}{1+x} d x\right) \\
& \stackrel{(g)}{\approx} \frac{1}{2} \log _{2}(e)\left(\int_{0}^{\frac{1}{N_{A}}} \frac{1-N_{A} x}{1+x} d x+\int_{0}^{\frac{1}{N_{B}}} \frac{1-N_{B} x}{1+x} d x\right) \\
& =\frac{1}{2} \log _{2}(e)\left[\left(1+N_{A}\right) \ln \left(\frac{1}{N_{A}}+1\right)+\left(1+N_{B}\right) \ln \left(\frac{1}{N_{B}}+1\right)-2\right],
\end{aligned}
$$

where in $(f)$ we use [23, Eq. (38)] and in $(g)$ we use (18). In addition, in $(g)$ we truncate the infinite upper limit of the two integrals due to the fact that we are only interested in the range of values for which $F_{A}(x), F_{B}(x) \leq 1$.

\section{Insights Based on the Analytical Model}

Since this work is focused on the effect of imperfect channel estimates and PPP-based interference on the performance metrics of the two-way relaying channel with ANC, it is interesting to examine whether some insights can be acquired from the analytical expressions regarding how these imperfect estimates and this type of interference affect these metrics. Towards this, we examine the individual average error probabilities of sources $k$ and $\bar{k}$ and we consider the following two scenarios: i) $\sigma_{e_{h_{k}}}<\sigma_{e_{h_{\bar{k}}}}$ and $d_{k}=d_{\bar{k}}$, $P_{k}=P_{\bar{k}}=P_{R}, \sigma_{h_{k}}=\sigma_{h_{\bar{k}}}$. ii) $d_{k}<d_{\bar{k}}$ and $P_{k}=P_{\bar{k}}=P_{R}, \sigma_{h_{k}}=\sigma_{h_{\bar{k}}}, \sigma_{e_{h_{k}}}=\sigma_{e_{h_{\bar{k}}}}$. Scenario i) shows the impact of the imperfect channel estimates, whereas scenario ii) shows the impact of the PPP-based interference.

\section{Scenario i):}

Proposition 4. In scenario i), it holds that $P_{E_{k}}<P_{E_{\bar{k}}}$.

Proof: By hypothesis testing and by considering that $E_{I}\left\{I_{k}\right\}=E_{I}\left\{I_{\bar{k}}\right\}$ since $d_{k}=d_{\bar{k}}$, we have

$$
P_{E_{k}}<P_{E_{\bar{k}}} \stackrel{(h)}{\Rightarrow} N_{k}<N_{\bar{k}} \stackrel{(i)}{\Rightarrow} \sigma_{e_{h_{k}}}<\sigma_{e_{h_{\bar{k}}}}
$$


which is true. In $(h)$ we use (26) and (29), whereas in $(i)$ we use (19).

Scenario i) simply tells us that if the variance of the estimation error is higher in the source $\bar{k}$-relay link than the one of the source $k$-relay link and all the other parameters of the system are the same for both links, then source $\bar{k}$ exhibits a higher average SER than the average SER of source $k$.

Scenario ii): To make the effect of PPP-based interference clearer, we consider two cases: a) There is no network interference, which means that $I_{R}=I_{k}=I_{\bar{k}}=0$. b) There is network interference affecting both the relay and the two sources, which means that $I_{R}, I_{k}, I_{\bar{k}} \neq 0$.

Proposition 5. In scenario ii), it holds that $P_{E_{k}}>P_{E_{\bar{k}}}$ for case a), whereas no conclusion can be drawn for case $b)$.

Proof: For case a), by hypothesis testing and considering that $I_{R}=I_{k}=I_{\bar{k}}=0$, we have

$$
P_{E_{k}}>P_{E_{\bar{k}}} \stackrel{(h)}{\Rightarrow} N_{k}>N_{\bar{k}} \stackrel{(i)}{\Rightarrow} d_{k}<d_{\bar{k}}
$$

which is true. $(h)$ and $(i)$ are defined in Proposition 4. The reason why this happens is because the amplified noise at the relay is attenuated less in the source $k$-relay link than the source $\bar{k}$-relay link, since $d_{k}<d_{\bar{k}}$. Hence, its effect is more significant at source $k$ than source $\bar{k}$.

For case b), no definite conclusion can be drawn regarding the relation between $P_{E_{k}}$ and $P_{E_{\bar{k}}}$ based on the analytical expressions. Intuitively, this relation totally depends on the position of sources $k$ and $\bar{k}$ and the values of the rest of the parameters. This is explained by the fact that although the amplified noise and the network interference affecting the relay are attenuated less in the source $k$-relay link than the source $\bar{k}$-relay link, if source $\bar{k}$ is close to the border of the exclusion region the total effect of interference plus noise might be greater at source $\bar{k}$ than source $k$, which is verified in Section IV.

\section{NumericAl Results}

Our aim in this section is twofold: i) To validate the theoretical results regarding the average sum SER and the achievable sum rate of the system by comparing them against Monte Carlo-based simulations in symmetric and asymmetric scenarios. ii) To validate the proved insights of Section III-D.

The Monte Carlo simulations are obtained by simulating the whole communication system, such as the modulator, propagation channel, and the demodulator. For the generation of the network interference, we consider the following procedure: i) We consider a finite circular area of sufficiently large radius, which we denote as $R_{a}$, which results in interferers located close to the edge having a negligible effect on the 


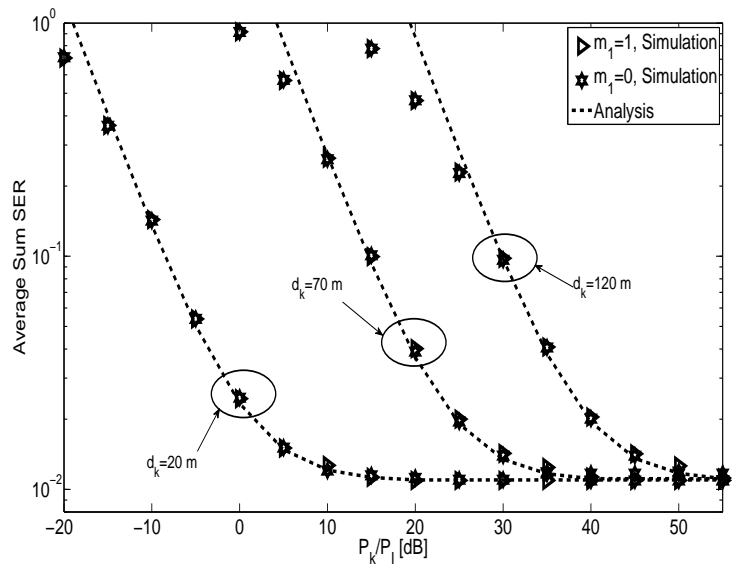

(a) $\sigma_{e_{h_{k}}}^{2}=0.001$.

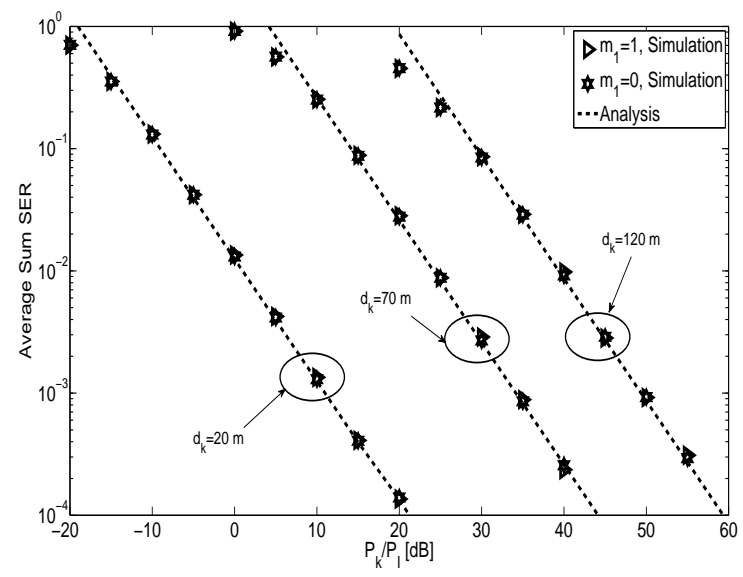

(b) $\sigma_{e_{h_{k}}}^{2}=0$.

Fig. 2: Average sum SER vs. $\frac{P_{k}}{P_{I}}[\mathrm{~dB}]$ for $a=4$.

relay and the two sources. In particular, we use $R_{a}=5000 \mathrm{~m}$. This way, we can model the effect of the theoretically infinite radius of the system; ii) The number of interferers for each of the three pair-wise independent PPPs, $i_{1}, i_{2}$, and $i_{3}$ is generated according to a Poisson distribution with densities $m_{1} \lambda, m_{2} \lambda$, and $m_{2} \lambda$, respectively, and area $\pi R_{a}^{2}$; iii) The locations of the interferers are distributed according to a uniform distribution in the circular region of area $\pi R_{a}^{2}$. Furthermore, we render inactive the nodes that are generated inside a circle with radius $R_{\text {exc }}$ (exclusion region of the interference); and iv) Independent complex channel coefficients are generated for each interferer. In addition, if $m_{1} \neq 0$, the same locations of the interferers that belong to the PPP $i_{1}$ are maintained in the simulator in the two time slots that the communication lasts and only the corresponding complex channel coefficients of the interferers change independently in these two slots. This is how $i_{1_{R}}, i_{1_{A}}$, and $i_{1_{B}}$ are obtained.

In addition, for the cases where $a \neq 2 m, m=2,3,4, \ldots$, and $R_{e x c}>(3+2 \sqrt{2}) d_{k}$, the second case from (23) holds, where the first series grows to infinity. For those cases, we use the first 15 terms of the infinite series in those analytical expressions. For all the obtained results we used $P_{I} / N_{0}=100 \mathrm{~dB}$, where $N_{0}=1$ is the variance of the AWGN affecting the relay and the two sources, $\lambda=10^{-5}\left(\frac{\text { nodes }}{m^{2}}\right)$, and MQAM modulation with $M=4$.

\section{A. Symmetric Scenario}

We consider the following setup: $R_{e x c}=150 \mathrm{~m}, d_{A}=d_{B}=d_{k}, P_{A}=P_{B}=P_{R}=P_{k}, \sigma_{e_{h_{A}}}^{2}=\sigma_{e_{h_{B}}}^{2}=$ $\sigma_{e_{h_{k}}}^{2}$, and $\sigma_{h_{A}}^{2}=\sigma_{h_{B}}^{2}=1$. 


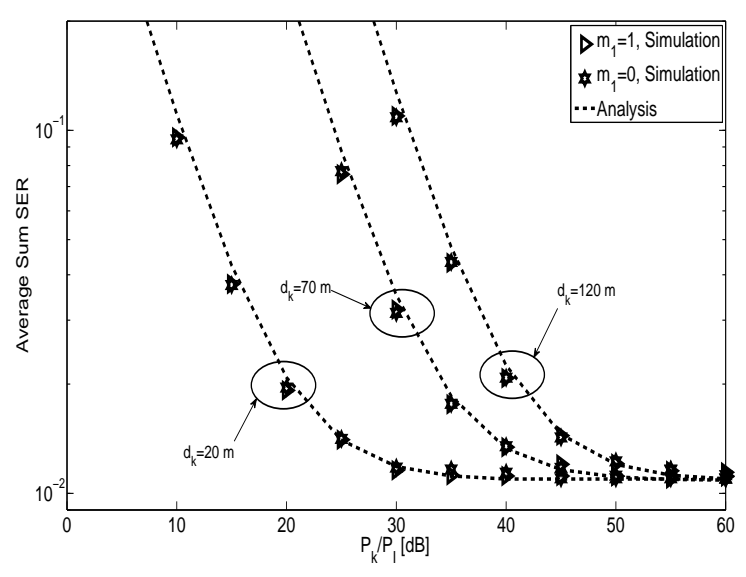

(a) $\sigma_{e_{h_{k}}}^{2}=0.001$.

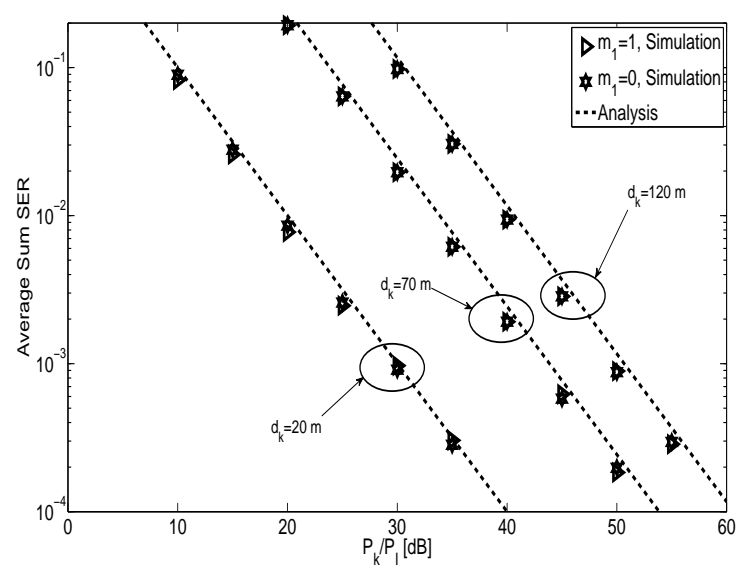

(b) $\sigma_{e_{h_{k}}}^{2}=0$.

Fig. 3: Average sum SER vs. $\frac{P_{k}}{P_{I}}[\mathrm{~dB}]$ for $a=2.5$.

1) Average Sum SER: In Fig. 2, we illustrate the average sum SER vs. $\frac{P_{k}}{P_{I}}$ plots for $a=4$, two values of $\sigma_{e_{h_{k}}}^{2}$, three values of $d_{k}$, and for $m_{1}=1$ and $m_{1}=0$. The $m_{1}=1$ case corresponds to the scenario where the same interferers are active at both time slots with their location being the same, whereas in the $m_{1}=0$ case the sets of interferers that are active at two time slots are disjoint. As we observe from Fig. 2, for all cases there is a good match of the theoretical analysis with the simulation results for error rates lower than 0.1 (high-SINR region), which substantiates the significance of the derived analytical error rate framework. In addition, we observe that the simulation plots for the $m_{1}=1$ and $m_{1}=0$ cases overlap in that region, which numerically validates the based on the theoretical analysis Remark 1. Furthermore, we observe that there is also an overlap in the low and medium-SINR region. This non-dependence of the performance metrics on the amount of correlation between the interference processes that affect the relay and the two sources can intuitively be justified by the fact that the two sources are unaware of the interference and, moreover, no combining is used ${ }^{5}$. Moreover, we see that the average sum SER reduces as $d_{k}$ increases, which was expected due to the fact that stronger interference affects the two sources for increasing $d_{k}$ due to the smaller distances between the interferers and the two sources.

In Fig. 3, we provide the same plots as with Fig. 2 with the only difference that we consider $a=2.5$. We again observe the good match of the theoretical with the simulation results for error rates lower than

\footnotetext{
${ }^{5}$ Such findings are similar to the ones that can be inferred by observing Figs. 2 and 3 of [34], which illustrate the average symbol error probability for an interference-unaware receiver in the cases of full correlation and independence between the two interference processes affecting the relay and the destination. By careful observation of these two figures, we can see that the simulated error probabilities in the two cases of full correlation and independence are the same, which means that correlation does not have an effect on the performance metrics. On the other hand, these error probabilities differ with respect to each other for an interference-aware receiver, as it can be observed in Figs. 4 and 5 of [34].
} 


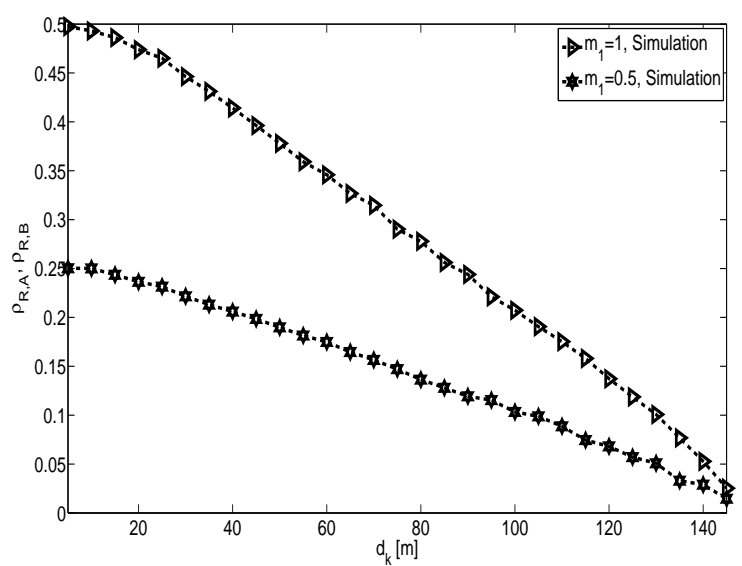

(a) $a=4$.

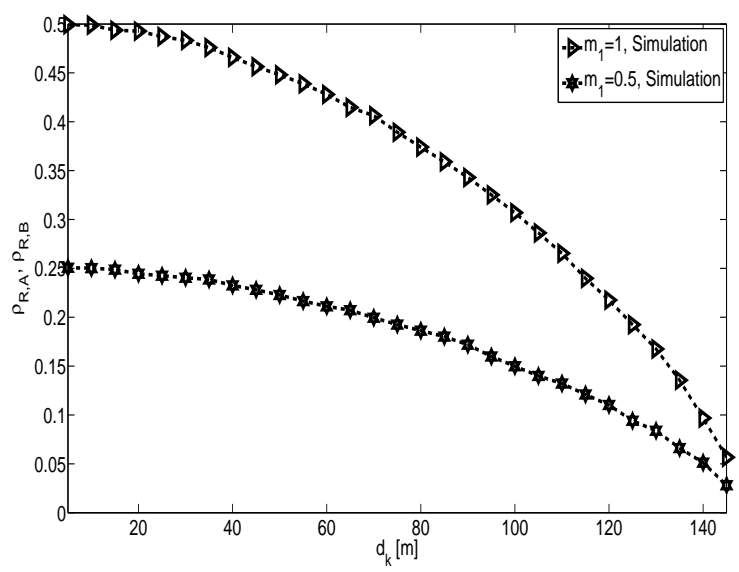

(b) $a=2.5$.

Fig. 4: $\rho_{R, A}, \rho_{R, B}$ vs. $d_{k}$.

0.1 together with the overlap in all the SINR range of the $m_{1}=1$ and $m_{1}=0$ cases.

Now, to numerically quantify the correlation between the interference processes that affect the relay and the two sources, in Fig. 4 we illustrate the $\rho_{R, A}, \rho_{R, B}$ vs. $d_{k}$ plots, where $\rho_{R, A}, \rho_{R, B}$ have been obtained by means of Monte Carlo simulations, according to (11). As we observe from Fig. 4, the correlation coefficient decreases as $d_{k}$ increases, which is attributed to the fact that the closer the sources are to the relay, the closer the distances between the interferers and the relay and between the interferers and the sources are with respect to each other. In addition, although for small values of $d_{k}$ the distances between the interferers and the relay and between the interferers and the two sources are almost equal, in Fig. 4 we also observe that $\rho_{R, A}$ and $\rho_{R, B}$ are not higher than 0.5 for small values of $d_{k}$, which is due to the independent channel fading in the two time slots that the communication lasts. Finally, we also observe that the values of $\rho_{R, A}$ and $\rho_{R, B}$ are higher for $a=2.5$ than for $a=4$, which can be attributed to less severe attenuation of the interfering signals as $a$ decreases.

2) Average Sum Rate: In Fig. 5 and Fig. 6, we illustrate the average sum rate vs. $\frac{P_{k}}{P_{I}}$ plots for the same configurations as with Fig. 2 and Fig. 3, respectively. We again observe a relatively good match in the high-SINR region of the simulation with the analysis together with the overlap of the $m_{1}=1$ and $m_{1}=0$ cases for all the SINR range. Furthermore, we observe a non-negligible gap between the simulation and the analysis in the low and medium-SINR region, which was expected since the theoretical expression of the average sum rate of (32) is obtained by using the high-SINR approximation of $F_{A}(x)$ and $F_{B}(x)$, according to (18). 


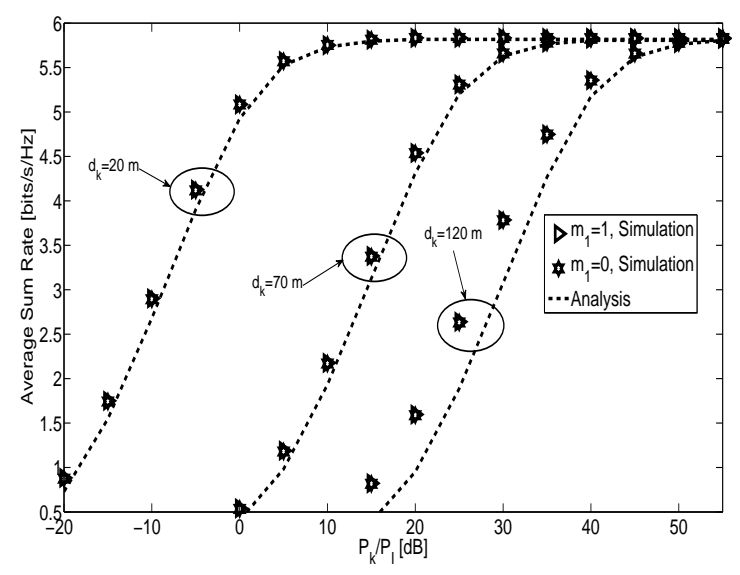

(a) $\sigma_{e_{h_{k}}}^{2}=0.001$.

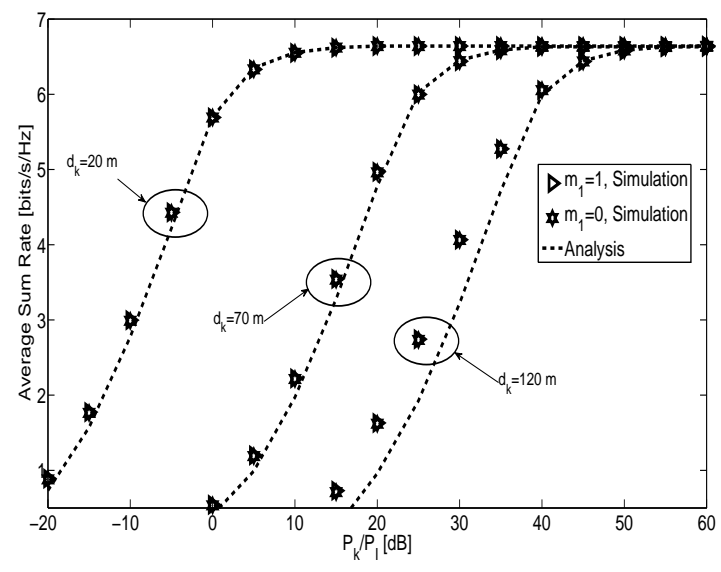

(b) $\sigma_{e_{h_{k}}}^{2}=0$.

Fig. 5: Average sum rate vs. $\frac{P_{k}}{P_{I}}[\mathrm{~dB}]$ for $a=2.5$.

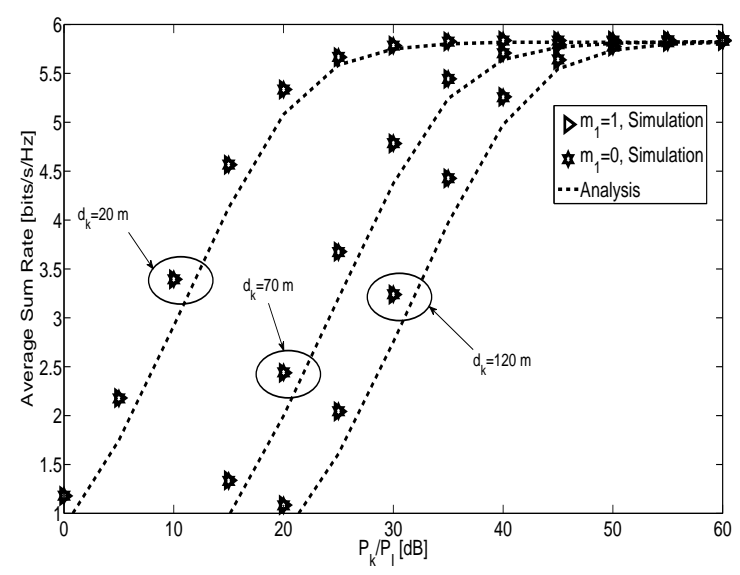

(a) $\sigma_{e_{h_{k}}}^{2}=0.001$.

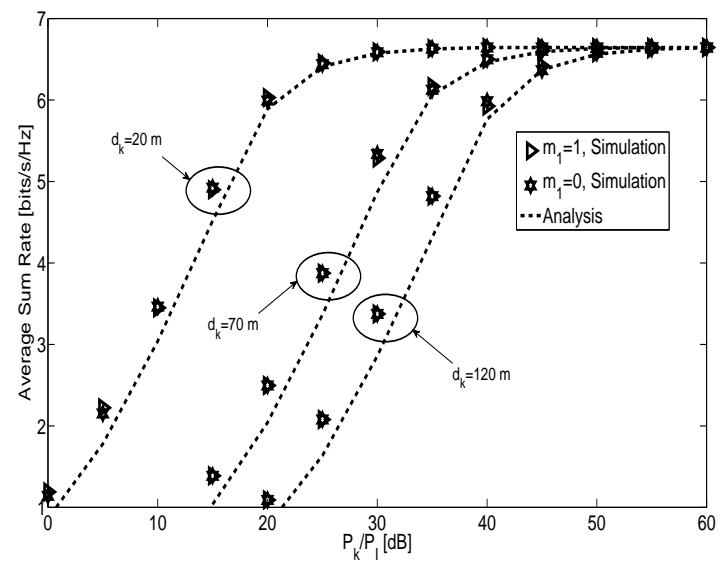

(b) $\sigma_{e_{h_{k}}}^{2}=0$.

Fig. 6: Average sum rate vs. $\frac{P_{k}}{P_{I}}[\mathrm{~dB}]$ for $a=2.5$.

\section{B. Asymmetric Scenario}

To also show the flexibility of the analytical expressions regardless of the value of the parameters, in

Fig. 7 we illustrate the average sum SER and sum rate vs. $\frac{P_{A}}{P_{I}}$ plots, respectively, for an asymmetric scenario with $R_{e x c}=150 \mathrm{~m}, P_{B}=P_{R}=2 P_{A}, d_{A}=50 \mathrm{~m}, \sigma_{h_{A}}^{2}=1, \sigma_{h_{B}}^{2}=2, \sigma_{e_{h_{A}}}^{2}=10^{-5}, \sigma_{e_{h_{B}}}^{2}=5 \cdot 10^{-5}$, $a=3.2, m_{1}=0.5$, and three values of $d_{B}$. Again, we observe the good match in the high-SINR region $\left(\mathrm{SER}<10^{-1}\right)$ of the analytical model with the Monte Carlo simulations.

\section{Validation of the Insights of Section III-D}

In this section, we validate the proved insights of Section III-D. We first start by validating Proposition 4. Towards this, we consider the following setup: $R_{e x c}=200 \mathrm{~m}, d_{A}=d_{B}=d_{k}, P_{A}=P_{B}=P_{R}=P_{k}$, 


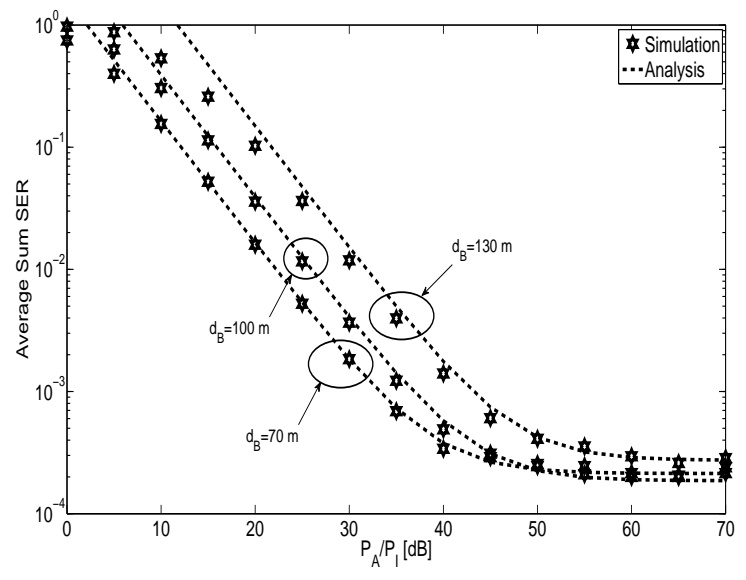

(a) SER.

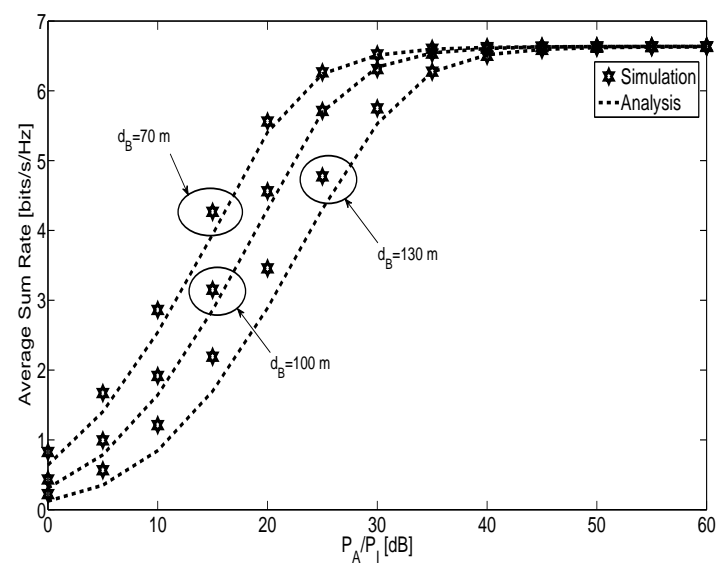

(b) Rate.

Fig. 7: Average sum SER and sum rate vs. $\frac{P_{A}}{P_{I}}[\mathrm{~dB}]$.

$\sigma_{h_{A}}^{2}=\sigma_{h_{B}}^{2}=1, \sigma_{e_{h_{A}}}^{2}=10^{-4}, \sigma_{e_{h_{B}}}^{2}=5 \cdot 10^{-4}, a=4$, and $m_{1}=0.5$. As we observe in Fig. 8, the higher value of the error variance of the source $B$-relay link compared to the corresponding one of the source $A$-relay link results in $P_{E_{B}}>P_{E_{A}}$ for two different values of $d_{k}$.

In addition, to validate Proposition 5 we consider the following setup: $R_{e x c}=200 \mathrm{~m}, d_{A}=50 \mathrm{~m}$, $P_{A}=P_{B}=P_{R}=P_{k}, \sigma_{h_{A}}^{2}=\sigma_{h_{B}}^{2}=1, \sigma_{e_{h_{A}}}^{2}=\sigma_{e_{h_{B}}}^{2}=0, a=4$, and $m_{1}=0.5$. In Fig. 9 (a), we consider that there is no interference and we illustrate the $P_{E_{A}}, P_{E_{B}}$ curves vs. $P_{k} / N_{0}$. As we observe, $P_{E_{A}}>P_{E_{B}}$ for both values of $d_{B}$ that are greater than $d_{A}$ and this is justified by the fact, as we aforementioned in Section III-D, that due to the higher distance of source $B$ to the relay the amplified noise is attenuated more in that link than in the source $A$-relay link. On the other hand, by including interference we observe in Fig. 9 (b) that $P_{E_{A}}>P_{E_{B}}$ for $d_{B}=80 \mathrm{~m}$, whereas $P_{E_{A}}<P_{E_{B}}$ for $d_{B}=130 \mathrm{~m}$. This is justified by the fact that in the case of $d_{B}=130 \mathrm{~m}$ the interference affecting source $B$ is quite strong since source $B$ is close to the border of the exclusion region, which eliminates the advantage of higher attenuation of the amplified noise plus interference at the relay due to the higher distance of the source $B$-relay link than the one of the source $A$-relay link. Hence, from Fig. 9 (b) we understand how important it is to accurately model the spatial characteristics of interference. Such distance-based trends cannot be captured by the Gaussian assumption for the interference since that model does not incorporate the distance of the interferers to the affected nodes. 


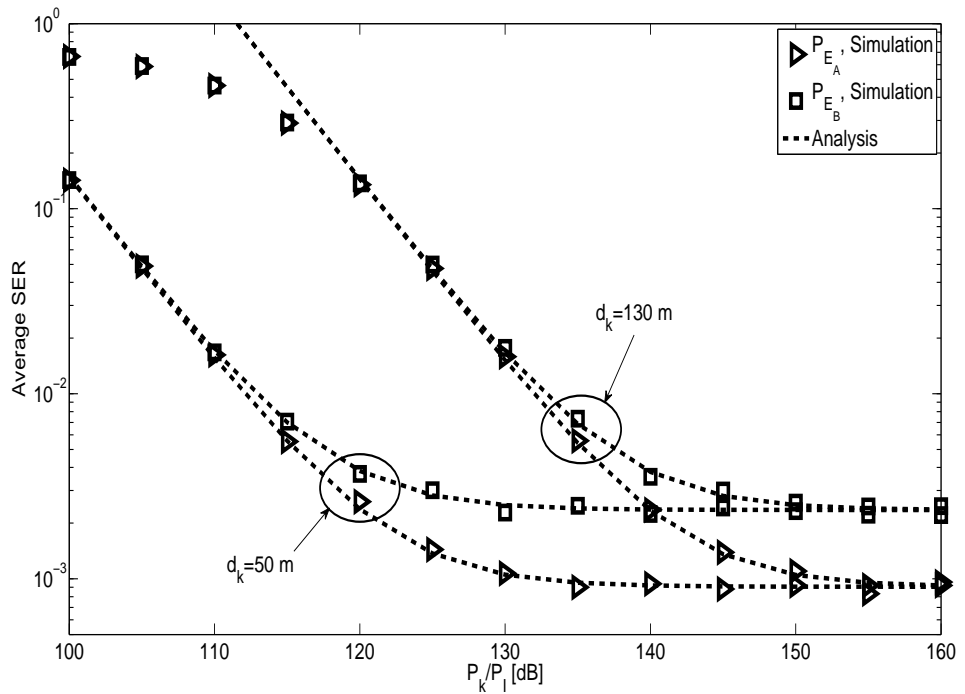

Fig. 8: Average SER vs. $\frac{P_{k}}{P_{I}}[\mathrm{~dB}]$.

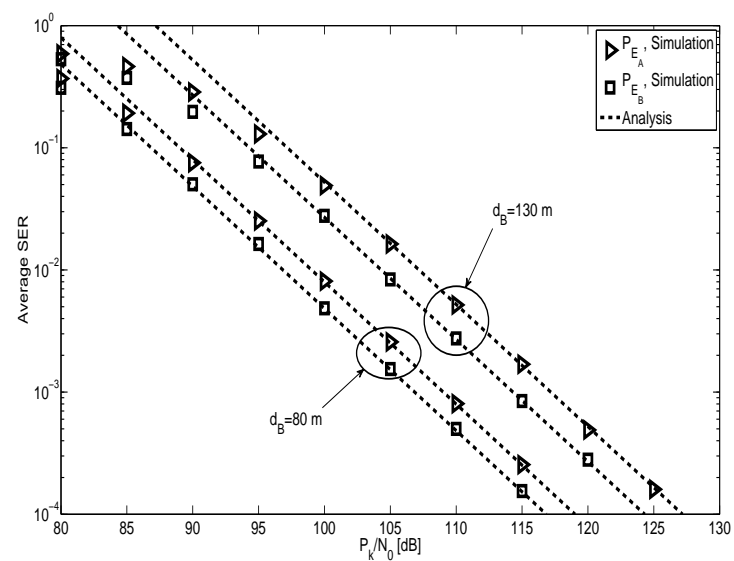

(a) Without interference.

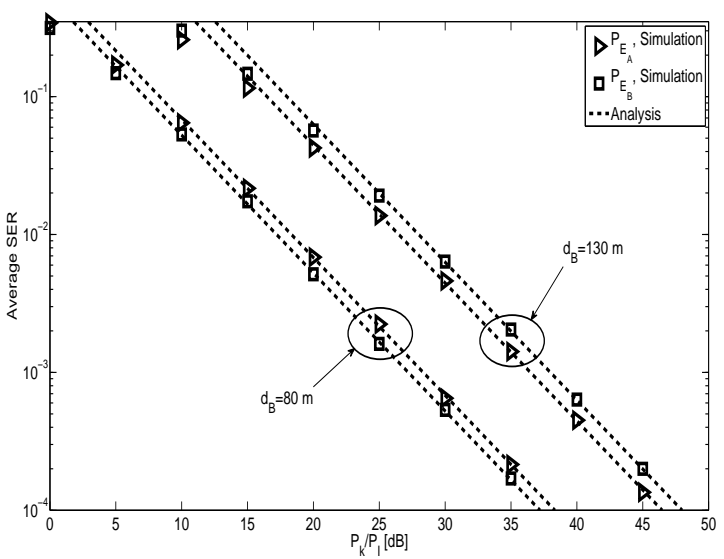

(b) With interference.

Fig. 9: Average SER vs. (a) $\frac{P_{k}}{N_{0}}[\mathrm{~dB}]$ and (b) $\frac{P_{k}}{P_{I}}[\mathrm{~dB}]$.

\section{Concluding Remarks And Future Work}

In this paper, we have investigated the joint effect of channel estimation errors and network interference on the performance of two-way relaying systems with ANC. In particular, a general model for the interference processes that affect the relay and the two sources has been presented, which incorporates the expected correlation between them. Subsequently, after deriving the statistics of the SINR of the two sources, we provided high-SINR analytical expressions of the system average sum SER and achievable average sum rate, which exhibit a relatively good match with the simulation results in the specific region. Furthermore, important insights regarding the impact of the imperfect channel estimates and network interference on the performance of the system were gained based on the analytical expressions. 
In addition, the theoretical analysis reveals that the amount of correlation between the interference processes that affect the relay and the two sources does not affect the examined performance metrics in the high-SINR region. This trend is validated by simulations, which also show that for the whole SINR range the amount of correlation does not affect these metrics.

Future work will focus on the study of the multiple-antenna relay case, which is expected to provide diversity gains. In addition, the study of the full-duplex relay case is another topic of interest.

\section{APPENDIX}

\section{A. Proof of Proposition 1}

We are going to prove that the statistical effect of the random terms $G_{R} \frac{\sqrt{P_{k}}}{d_{k}^{a}} e_{h_{k}}^{2} s_{k}, G_{R} \sqrt{\frac{P_{\bar{k}}}{d_{k}^{a} d_{\bar{k}}^{a}}} e_{h_{k}} e_{h_{\bar{k}}}$, and $G_{R} \frac{n_{R} e_{h_{k}}}{\sqrt{d_{k}^{a}}}$ of (6) is negligible since their second moment (average power effect) is much smaller than the second moment of other terms of this equation. More specifically,

i) We first consider the term $G_{R} \frac{\sqrt{P_{k}}}{d_{k}^{a}} e_{h_{k}}^{2} s_{k}$ of (6) and let us take the ratio its second moment with the corresponding one of the term $2 G_{R} \frac{\sqrt{P_{k}}}{d_{k}^{a}} \hat{h}_{k} e_{h_{k}} s_{k}$ of the same equation. We have

$$
\frac{E\left\{\left|G_{R} \frac{\sqrt{P_{k}}}{d_{k}^{a}} e_{h_{k}}^{2} s_{k}\right|^{2}\right\}}{E\left\{\left|2 G_{R} \frac{\sqrt{P_{k}}}{d_{k}^{a}} \hat{h}_{k} e_{h_{k}} s_{k}\right|^{2}\right\}}=\frac{3 \sigma_{e_{h_{k}}}^{4}}{4\left(\sigma_{h_{k}}^{2}-\sigma_{e_{h_{k}}}^{2}\right) \sigma_{e_{h_{k}}}^{2}}=\frac{3 \sigma_{e_{h_{k}}}^{2}}{4\left(\sigma_{h_{k}}^{2}-\sigma_{e_{h_{k}}}^{2}\right)} \ll 1,
$$

for $\sigma_{h_{k}} \gg \sigma_{e_{h_{k}}}$, which occurs in practical systems. Hence, the term $G_{R} \frac{\sqrt{P_{k}}}{d_{k}^{a}} e_{h_{k}}^{2} s_{k}$ can be eliminated from (6) since its statistical power effect is negligible.

ii) We now consider the ratio of the second moment of the term $G_{R} \sqrt{\frac{P_{\bar{k}}}{d_{k}^{a} d_{\bar{k}}^{a}}} e_{h_{k}} e_{h_{\bar{k}}}$ with the corresponding one of the term $G_{R} \sqrt{\frac{P_{\bar{k}}}{d_{k}^{a} d_{\bar{k}}^{a}}} \hat{h}_{k} e_{h_{k}} s_{\bar{k}}$ of (6). We have

$$
\frac{E\left\{\left|G_{R} \sqrt{\frac{P_{\bar{k}}}{d_{k}^{a} d_{\bar{k}}^{a}}} e_{h_{k}} e_{h_{\bar{k}}}\right|^{2}\right\}}{E\left\{\left|G_{R} \sqrt{\frac{P_{\bar{k}}}{d_{k}^{a} d_{\bar{k}}^{a}}} \hat{h}_{k} e_{h_{k}} s_{\bar{k}}\right|^{2}\right\}}=\frac{\sigma_{e_{h_{k}}}^{2} \sigma_{e_{h_{\bar{k}}}}^{2}}{\left(\sigma_{h_{k}}^{2}-\sigma_{e_{h_{k}}}^{2}\right) \sigma_{e_{h_{k}}}^{2}\left|s_{\bar{k}}\right|^{2}}=\frac{\sigma_{e_{h_{\bar{k}}}}^{2}}{\left(\sigma_{h_{k}}^{2}-\sigma_{e_{h_{k}}}^{2}\right)\left|s_{\bar{k}}\right|^{2}} \ll 1
$$

for typical modulation orders and $\sigma_{h_{k}} \gg \sigma_{e_{h_{k}}}, \sigma_{e_{h_{\bar{k}}}}$. Consequently, also the term $G_{R} \sqrt{\frac{P_{\bar{k}}}{d_{k}^{a} d_{\bar{k}}^{a}}} e_{h_{k}} e_{h_{\bar{k}}}$ can be eliminated from (6) since its statistical power effect is negligible.

iii) Finally, we consider the ratio of the second moment of the term $G_{R} \frac{n_{R} e_{h_{k}}}{\sqrt{d_{k}^{a}}}$ with the corresponding 
one of the term $G_{R} \frac{n_{R} \hat{h}_{k}}{\sqrt{d_{k}^{a}}}$ of (6). We have

$$
\frac{E\left\{\left|G_{R} \frac{n_{R} e_{h_{k}}}{\sqrt{d_{k}^{a}}}\right|^{2}\right\}}{E\left\{\left|G_{R} \frac{n_{R} \hat{h}_{k}}{\sqrt{d_{k}^{a}}}\right|^{2}\right\}}=\frac{\sigma_{e_{h_{k}}}^{2}}{\sigma_{h_{k}}^{2}-\sigma_{e_{h_{k}}}^{2}} \ll 1,
$$

for $\sigma_{h_{k}} \gg \sigma_{e_{h_{k}}}$. Hence, the term $G_{R} \frac{n_{R} e_{h_{k}}}{\sqrt{d_{k}^{a}}}$ can be eliminated from (6).

\section{B. Proof of Proposition 2}

We are going to prove that the average value of each of the terms $\frac{P_{R} I_{R} \sigma_{e_{h_{k}}}^{2}}{d_{k}^{a}}, \frac{P_{k}}{d_{k}^{a}} \sigma_{e_{h_{k}}}^{2}+\frac{P_{\bar{k}}}{d_{\bar{k}}^{a}} \sigma_{e_{h_{\bar{k}}}}^{2}$, and $I_{k}\left(\frac{P_{k}}{d_{k}^{a}} \sigma_{e_{h_{k}}}^{2}+\frac{P_{\bar{k}}}{d_{\bar{k}}^{a}} \sigma_{e_{h_{\bar{k}}}}^{2}\right)$ of $\mu$ is much smaller than the average value of other terms of the denominator of (14) and, hence, the statistical effect of $\mu$ is negligible. More specifically,

i) We consider the term $\frac{P_{R} I_{R} \sigma_{e_{h_{k}}}^{2}}{d_{k}^{a}}$ and take the ratio of its average value with the average value of the term $\frac{P_{R} I_{R}}{d_{k}^{a}}\left|\hat{h}_{k}\right|^{2}$ in the denominator of (14). We have

$$
\frac{E\left\{\frac{P_{R} I_{R} \sigma_{e_{h_{k}}}^{2}}{d_{k}^{a}}\right\}}{E\left\{\frac{P_{R} I_{R}}{d_{k}^{a}}\left|\hat{h}_{k}\right|^{2}\right\}}=\frac{\sigma_{e_{h_{k}}}^{2}}{\sigma_{h_{k}}^{2}-\sigma_{e_{h_{k}}}^{2}} \ll 1 .
$$

Hence, the statistical effect of $\frac{P_{R} I_{R} \sigma_{e_{h_{k}}}^{2}}{d_{k}^{a}}$ is negligible.

ii) We now consider the ratio of the average value of the term $\frac{P_{k}}{d_{k}^{a}} \sigma_{e_{h_{k}}}^{2}+\frac{P_{\bar{k}}}{d_{\bar{k}}^{a}} \sigma_{e_{h_{\bar{k}}}}^{2}$ with the corresponding one of the term $\frac{P_{k}\left|\hat{h}_{k}\right|^{2}}{d_{k}^{a}}+\frac{P_{\bar{k}}\left|\hat{h}_{\bar{k}}\right|^{2}}{d_{\bar{k}}^{a}}$ in the denominator of (14). We have

$$
\frac{E\left\{\frac{P_{k}}{d_{k}^{a}} \sigma_{e_{h_{k}}}^{2}+\frac{P_{\bar{k}}}{d_{\bar{k}}^{a}} \sigma_{e_{h_{\bar{k}}}}^{2}\right\}}{E\left\{\frac{P_{k}\left|\hat{h}_{k}\right|^{2}}{d_{k}^{a}}+\frac{P_{\bar{k}}\left|\hat{h}_{\bar{k}}\right|^{2}}{d_{\bar{k}}^{a}}\right\}}=\frac{\frac{P_{k}}{d_{k}^{a}} \sigma_{e_{h_{k}}}^{2}+\frac{P_{\bar{k}}}{d_{\bar{k}}^{a}} \sigma_{e_{h_{\bar{k}}}}^{2}}{\frac{P_{k}}{d_{k}^{a}}\left(\sigma_{h_{k}}^{2}-\sigma_{e_{h_{k}}}^{2}\right)+\frac{P_{\bar{k}}}{d_{\bar{k}}^{a}}\left(\sigma_{h_{\bar{k}}}^{2}-\sigma_{e_{h_{\bar{k}}}^{2}}^{2}\right)} \ll 1,
$$

since $\sigma_{e_{h_{k}}}, \sigma_{e_{h_{\bar{k}}}} \ll \sigma_{h_{k}}, \sigma_{h_{\bar{k}}}$. Consequently, also the statistical effect of $\frac{P_{k}}{d_{k}^{a}} \sigma_{e_{h_{k}}}^{2}+\frac{P_{\bar{k}}}{d_{\bar{k}}^{a}} \sigma_{e_{h_{\bar{k}}}}^{2}$ is negligible.

iii) Finally, we consider the ratio of the average value of the term $I_{k}\left(\frac{P_{k}}{d_{k}^{a}} \sigma_{e_{h_{k}}}^{2}+\frac{P_{\bar{k}}}{d_{\bar{k}}^{a}} \sigma_{e_{h_{\bar{k}}}}^{2}\right)$ with the corresponding one of the term $I_{k}\left(\frac{P_{k}\left|\hat{h}_{k}\right|^{2}}{d_{k}^{a}}+\frac{P_{\bar{k}}\left|\hat{h}_{\bar{k}}\right|^{2}}{d_{\bar{k}}^{a}}\right)$ in the denominator of (14). We have

$$
\frac{E\left\{I_{k}\left(\frac{P_{k}}{d_{k}^{a}} \sigma_{e_{h_{k}}}^{2}+\frac{P_{\bar{k}}}{d_{\bar{k}}^{a}} \sigma_{e_{h_{\bar{k}}}}^{2}\right)\right\}}{E\left\{I_{k}\left(\frac{P_{k}\left|\hat{h}_{k}\right|^{2}}{d_{k}^{a}}+\frac{P_{\bar{k}}\left|\hat{h}_{\bar{k}}\right|^{2}}{d_{\bar{k}}^{\bar{a}}}\right)\right\}}=\frac{\frac{P_{k}}{d_{k}^{a}} \sigma_{e_{h_{k}}}^{2}+\frac{P_{\bar{k}}}{d_{\bar{k}}^{a}} \sigma_{e_{h_{\bar{k}}}}^{2}}{\frac{P_{k}}{d_{k}^{a}}\left(\sigma_{h_{k}}^{2}-\sigma_{e_{h_{k}}}^{2}\right)+\frac{P_{\bar{k}}}{d_{\bar{k}}^{a}}\left(\sigma_{h_{\bar{k}}}^{2}-\sigma_{e_{h_{\bar{k}}}}^{2}\right)} \ll 1 .
$$


Hence, the statistical effect of $I_{k}\left(\frac{P_{k}}{d_{k}^{a}} \sigma_{e_{h_{k}}}^{2}+\frac{P_{\bar{k}}}{d_{\bar{k}}^{a}} \sigma_{e_{h_{\bar{k}}}}^{2}\right)$ is negligible.

\section{Proof of Proposition 3}

We derive $F_{k}(x)$ in a two-step procedure: i) We first derive $F_{k}(x)$ conditioned on the network interference (the statistics of the distances), which we denote as $F_{k}(x \mid I)$, by averaging over $\left|\hat{h}_{k}\right|^{2}$ and $\left|\hat{h}_{\bar{k}}\right|^{2}$. ii) Subsequently, we derive $F_{k}(x)$ by averaging over the statistics of the network interference.

i) $F_{k}(x \mid I)$ : We have that

$$
\begin{aligned}
F_{k}(x \mid I) & \stackrel{(a)}{=} \operatorname{Pr}\left(\frac{P_{\bar{k}} P_{R}\left|\hat{h}_{k}\right|^{2}\left|\hat{h}_{\bar{k}}\right|^{2}}{C_{k}\left|\hat{h}_{k}\right|^{2}+D_{k}\left|\hat{h}_{\bar{k}}\right|^{2}} \leqslant x\right)=\operatorname{Pr}\left(\left|\hat{h}_{k}\right|^{2}\left(P_{\bar{k}} P_{R}\left|\hat{h}_{\bar{k}}\right|^{2}-C_{k} x\right) \leqslant D_{k}\left|\hat{h}_{\bar{k}}\right| x\right) \\
& =\operatorname{Pr}\left(P_{\bar{k}} P_{R}\left|\hat{h}_{\bar{k}}\right|^{2}-C_{k} x \leqslant 0\right)+\operatorname{Pr}\left(\left|\hat{h}_{k}\right|^{2} \leqslant\left.\frac{D_{k}\left|\hat{h}_{\bar{k}}\right| x}{P_{\bar{k}} P_{R}\left|\hat{h}_{\bar{k}}\right|^{2}-C_{k} x}\left|P_{\bar{k}} P_{R}\right| \hat{h}_{\bar{k}}\right|^{2}-C_{k} x>0\right)
\end{aligned}
$$

where in $(a)$ we use (15).

For the first term of (41), we have

$$
\operatorname{Pr}\left(P_{\bar{k}} P_{R}\left|\hat{h}_{\bar{k}}\right|^{2}-C_{k} x \leqslant 0\right)=\operatorname{Pr}\left(\left|\hat{h}_{\bar{k}}\right|^{2} \leqslant \frac{C_{k} x}{P_{\bar{k}} P_{R}}\right)=1-e^{\frac{C_{h_{\bar{k}}} x}{\left(\sigma_{h_{\bar{k}}}^{2}-\sigma_{e_{\bar{k}}}^{2}\right) P_{\bar{k}} P_{R}}} .
$$

For the second term of (41), we have

$$
\begin{aligned}
& \operatorname{Pr}\left(\left|\hat{h}_{k}\right|^{2} \leqslant\left.\frac{D_{k}\left|\hat{h}_{\bar{k}}\right| x}{P_{\bar{k}} P_{R}\left|\hat{h}_{\bar{k}}\right|^{2}-C_{k} x}\left|P_{\bar{k}} P_{R}\right| \hat{h}_{\bar{k}}\right|^{2}-C_{k} x>0\right)=\int_{\frac{C_{k} x}{P_{\bar{k}} P_{R}}}^{\infty}\left(1-e^{\left.-\frac{1}{\sigma_{h_{k}}^{2}-\sigma_{e_{h}}^{2} \frac{D_{k} y x}{P_{\bar{k}} P_{R} y-C_{k} x}}\right)}\right.
\end{aligned}
$$

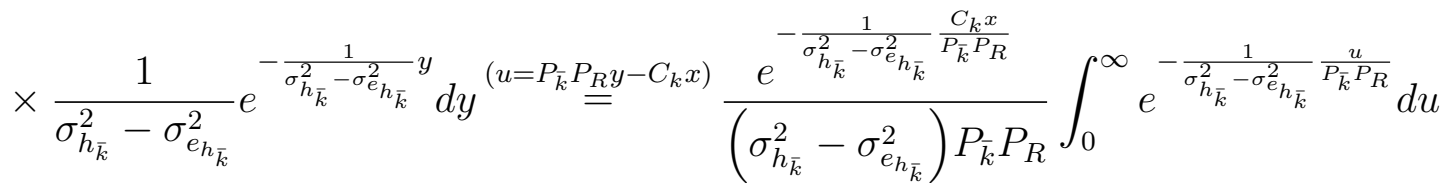

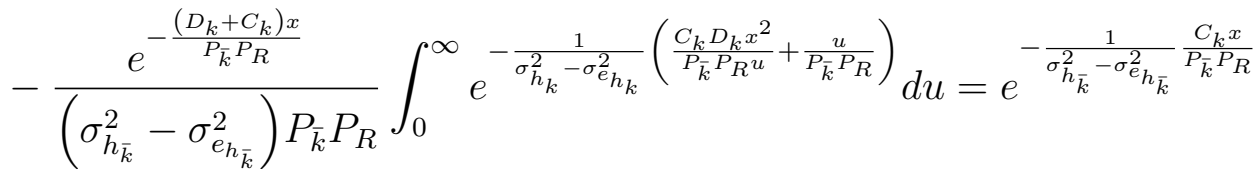

$$
\begin{aligned}
& -\frac{e^{-\left[\frac{1}{\sigma_{h_{\bar{k}}}^{2}-\sigma_{e_{\bar{h}}}^{2}} \frac{C_{k} x}{P_{\bar{k}} P_{R}}+\frac{1}{\sigma_{h_{k}}^{2}-\sigma_{e_{h_{k}}}^{2}} \frac{D_{k} x}{P_{\bar{k}} P_{R}}\right]}}{\left(\sigma_{h_{\bar{k}}}^{2}-\sigma_{e_{h_{\bar{k}}}}^{2}\right) P_{\bar{k}} P_{R}} 2 x \sqrt{\frac{\sigma_{h_{\bar{k}}}^{2}-\sigma_{e_{h_{\bar{k}}}}^{2}}{\sigma_{h_{k}}^{2}-\sigma_{e_{h_{k}}}^{2}} C_{k} D_{k}} \\
& \times K_{1}\left(\frac{2 x}{P_{\bar{k}} P_{R}} \sqrt{\frac{1}{\left(\sigma_{h_{k}}^{2}-\sigma_{e_{h_{k}}}^{2}\right)\left(\sigma_{h_{\bar{k}}}^{2}-\sigma_{e_{h_{\bar{k}}}}^{2}\right)} C_{k} D_{k}},\right.
\end{aligned}
$$


where the second term of (43) follows from [38, 3.478.4]. If $P_{\bar{k}}$ and $P_{R}$ have high enough values such that $\frac{2 x}{P_{\bar{k}} P_{R}} \sqrt{\frac{1}{\left(\sigma_{h_{k}}^{2}-\sigma_{e_{h_{k}}}^{2}\right)\left(\sigma_{h_{\bar{k}}}^{2}-\sigma_{e_{h_{\bar{k}}}^{2}}^{2}\right)} C_{k} D_{k}} \ll 1$ (high-SINR region), we can use the small argument approximation of $K_{1}(\cdot)$, i.e. $K_{1}(z) \approx \frac{1}{z}, z \ll 1$ [39]. Hence, (43) becomes

$$
\begin{aligned}
& \operatorname{Pr}\left(\left|\hat{h}_{k}\right|^{2} \leqslant\left.\frac{D_{k}\left|\hat{h}_{\bar{k}}\right| x}{P_{\bar{k}} P_{R}\left|\hat{h}_{\bar{k}}\right|^{2}-C_{k} x}\left|P_{\bar{k}} P_{R}\right| \hat{h}_{\bar{k}}\right|^{2}-C_{k} x>0\right) \approx e^{-\frac{C_{k} x}{\left(\sigma_{h_{\bar{k}}}^{2}-\sigma_{e_{\bar{k}}}^{2}\right) P_{\bar{k}} P_{R}}} \\
& -e^{-\left[\frac{C_{k} x}{\left(\sigma_{h_{\bar{k}}}^{2}-\sigma_{e_{h_{\bar{k}}}^{2}}^{2}\right) P_{\bar{k}} P_{R}}+\frac{D_{k} x}{\left(\sigma_{h_{k}}^{2}-\sigma_{e_{h_{k}}}^{2}\right) P_{\bar{k}} P_{R}}\right]}
\end{aligned}
$$

By substituting (42) and (44) into (41), we get

$$
F_{k}(x \mid I) \approx 1-e^{-\left[\frac{C_{k}}{\left(\sigma_{h_{\bar{k}}}^{2}-\sigma_{e_{\bar{k}}}^{2}\right) P_{\bar{k}} P_{R}}+\frac{D_{k}}{\left(\sigma_{h_{k}}^{2}-\sigma_{e_{h_{k}}}^{2}\right) P_{\bar{k}} P_{R}}\right] x} \stackrel{(b)}{\approx}\left[\frac{C_{k}}{\left(\sigma_{h_{\bar{k}}}^{2}-\sigma_{e_{\bar{k}}}^{2}\right) P_{\bar{k}} P_{R}}+\frac{D_{k}}{\left(\sigma_{h_{k}}^{2}-\sigma_{e_{h_{k}}}^{2}\right) P_{\bar{k}} P_{R}}\right] x,
$$

where in $(b)$ we have considered the small argument approximation $\left(e^{-x} \approx 1-x, x \ll 1\right)$ of the exponential function in (45) since the error probability in the high-SINR region is dominated by the behavior of the probability (or cumulative) density function (pdf) as $x$ (SINR) tends to 0 [40]. Hence, in the region of interest (high-SINR region) it holds that $\left[\frac{\left(\sigma_{h_{\bar{k}}}^{2}-\sigma_{e_{h_{\bar{k}}}^{2}}^{2}\right) C_{k}+\left(\sigma_{h_{k}}^{2}-\sigma_{e_{h_{k}}}^{2}\right) D_{k}}{P_{\bar{k}} P_{R}}\right] x \ll 1$ (the exponent in (45)).

ii) $F_{k}(x)$ : From (45), we have $F_{k}(x)=E_{I}\left\{F_{k}(x \mid I)\right\}$ and, hence, (19) is obtained from (41).

Now, we have that [41, Eq. (8)] (which is a result of Campbell's theorem for a PPP process [37])

$$
E_{I}\left\{I_{1_{R}}\right\}=\frac{2 \pi m_{1} \lambda P_{I}}{a-2} R_{e x c}^{2-a} \quad \text { and } \quad E_{I}\left\{I_{2}\right\}=\frac{2 \pi m_{2} \lambda P_{I}}{a-2} R_{\text {exc }}^{2-a}
$$

Furthermore, again according to Campbell's theorem, we have

$$
E_{I}\left\{I_{1_{k}}\right\}=m_{1} \lambda P_{I} \int_{R_{e x c}}^{\infty} l_{1} d l_{1} \int_{0}^{2 \pi} \frac{1}{\left(\sqrt{l_{1}^{2}+d_{k}^{2}-2 l_{1} d_{k} \cos \theta}\right)^{a}} d \theta
$$

By setting $l_{2}^{2}=l_{1}^{2}+d_{k}^{2}-2 l_{1} d_{k} \cos \theta$, we have

$$
\cos \theta=\frac{l_{1}^{2}+d_{k}^{2}-l_{2}^{2}}{2 l_{1} d_{k}} \Rightarrow \theta=\cos ^{-1}\left(\frac{l_{1}^{2}+d_{k}^{2}-l_{2}^{2}}{2 l_{1} d_{k}}\right) \Rightarrow d \theta=\frac{2 l_{2}}{\sqrt{l_{2}^{2}-\left(l_{1}^{2}-d_{k}^{2}\right)} \sqrt{\left(l_{1}^{2}+d_{k}^{2}\right)-l_{2}^{2}}} d l_{2} .
$$


By substituting (48) into (47), we get

$$
\begin{aligned}
& E_{I}\left\{I_{1_{k}}\right\}=2 m_{1} \lambda P_{I} \int_{R_{\text {exc }}}^{\infty} l_{1} d l_{1} \int_{l_{1}-d_{k}}^{l_{1}+d_{k}} l_{2}^{-a} \frac{2 l_{2}}{\sqrt{l_{2}^{2}-\left(l_{1}-d_{k}\right)^{2}} \sqrt{\left(l_{1}+d_{k}\right)^{2}-l_{2}^{2}}} d l_{2} \\
& \stackrel{t=l_{2}^{-2}}{=} 2 m_{1} \lambda P_{I} \int_{R_{e x c}}^{\infty} l_{1} d l_{1} \int_{\frac{1}{\left(l_{1}+d_{k}\right)^{2}}}^{\frac{1}{\left(l_{1}-d_{k}\right)^{2}}} \frac{t^{\frac{a}{2}-2}}{\sqrt{\frac{1}{t}-\left(l_{1}-d_{k}\right)^{2}} \sqrt{\left(l_{1}+d_{k}\right)^{2}-\frac{1}{t}}} d t \\
& =2 m_{1} \lambda P_{I} \int_{R_{e x c}}^{\infty} \frac{l_{1}}{\left(l_{1}-d_{k}\right)\left(l_{1}+d_{k}\right)} d l_{1} \int_{\frac{1}{\left(l_{1}+d_{k}\right)^{2}}}^{\frac{1}{\left(l_{1}-d_{k}\right)^{2}}} \frac{t^{\frac{a}{2}-1}}{\sqrt{\frac{1}{\left(l_{1}-d_{k}\right)^{2}}-t} \sqrt{t-\frac{1}{\left(l_{1}+d_{k}\right)^{2}}}} d t \\
& u=t-\frac{1}{\left(l_{1}+d_{k}\right)^{2}} 2 m_{1} \lambda P_{I} \int_{R_{e x c}}^{\infty} \frac{l_{1}}{\left(l_{1}-d_{k}\right)\left(l_{1}+d_{k}\right)} d l_{1} \\
& \times \int_{0}^{\frac{1}{\left(l_{1}-d_{k}\right)^{2}}-\frac{1}{\left(l_{1}+d_{k}\right)^{2}}} \frac{\left(u+\frac{1}{\left(l_{1}+d_{k}\right)^{2}}\right)^{\frac{a}{2}-1}}{\sqrt{u} \sqrt{\frac{1}{\left(l_{1}-d_{k}\right)^{2}}-\frac{1}{\left(l_{1}+d_{k}\right)^{2}}-u}} d u \text {. }
\end{aligned}
$$

Now, from (49) we distinguish two cases:

1) $a=2 m$, where $m=2,3,4, \ldots$. In this case, for the second integral of (49) we can take the binomial expansion of the numerator. This gives

$$
\begin{aligned}
& \int_{0}^{\frac{1}{\left(l_{1}-d_{k}\right)^{2}}-\frac{1}{\left(l_{1}+d_{k}\right)^{2}}} \frac{\left(u+\frac{1}{\left(l_{1}+d_{k}\right)^{2}}\right)^{\frac{a}{2}-1}}{\sqrt{u} \sqrt{\frac{1}{\left(l_{1}-d_{k}\right)^{2}}-\frac{1}{\left(l_{1}+d_{k}\right)^{2}}-u}} d u \\
& =\sum_{n=0}^{\frac{a}{2}-1}\left(\begin{array}{c}
\frac{a}{2}-1 \\
n
\end{array}\right)\left[\frac{1}{\left(l_{1}+d_{k}\right)^{2}}\right]^{\frac{a}{2}-1-n} \int_{0}^{\frac{1}{\left(l_{1}-d_{A}\right)^{2}}-\frac{1}{\left(l_{1}+d_{A}\right)^{2}}} \frac{u^{n-\frac{1}{2}}}{\sqrt{\frac{1}{\left(l_{1}-d_{k}\right)^{2}}-\frac{1}{\left(l_{1}+d_{k}\right)^{2}}-u}} d u \\
& =\sum_{n=0}^{\frac{a}{2}-1}\left(\begin{array}{c}
\frac{a}{2}-1 \\
n
\end{array}\right)\left[\frac{1}{\left(l_{1}+d_{k}\right)^{2}}\right]^{\frac{a}{2}-1-n}\left[\frac{4 l_{1} d_{k}}{\left(l_{1}-d_{k}\right)^{2}\left(l_{1}+d_{k}\right)^{2}}\right]^{n} B\left(\frac{1}{2}, n+\frac{1}{2}\right),
\end{aligned}
$$


which follows from [38, 3.191.1]. By substituting (50) into (49), we get

$$
\begin{aligned}
E_{I}\left\{I_{1_{k}}\right\} & =2 m_{1} \lambda P_{I} \sum_{n=0}^{\frac{a}{2}-1}\left(\begin{array}{c}
\frac{a}{2}-1 \\
n
\end{array}\right) B\left(\frac{1}{2}, n+\frac{1}{2}\right)\left(4 d_{k}\right)^{n} \int_{R_{e x c}}^{\infty} \frac{l_{1}^{n+1}}{\left(l_{1}-d_{k}\right)^{2 n+1}\left(l_{1}+d_{k}\right)^{a-1}} d l_{1} \\
& \stackrel{p=l_{1}-R_{e x c}}{=} 2 m_{1} \lambda P_{I} \sum_{n=0}^{\frac{a}{2}-1}\left(\begin{array}{c}
\frac{a}{2}-1 \\
n
\end{array}\right) B\left(\frac{1}{2}, n+\frac{1}{2}\right)\left(4 d_{k}\right)^{n} \\
& \times \int_{0}^{\infty} \frac{\left(p+R_{e x c}\right)^{n+1}}{\left(p+R_{e x c}-d_{k}\right)^{2 n+1}\left(p+R_{e x c}+d_{k}\right)^{a-1}} d p \\
& =2 m_{1} \lambda P_{I} \sum_{n=0}^{\frac{a}{2}-1}\left(\begin{array}{c}
\frac{a}{2}-1 \\
n
\end{array}\right) B\left(\frac{1}{2}, n+\frac{1}{2}\right)\left(4 d_{k}\right)^{n} \\
& \times \sum_{w=0}^{n+1}\left(\begin{array}{c}
n+1 \\
w
\end{array}\right) B\left(\frac{1}{2}, n+\frac{1}{2}\right)\left(4 d_{k}\right)^{n} \sum_{w=0}^{n+1}\left(\begin{array}{c}
n+1-w \\
\text { exc }
\end{array} \int_{0}^{\infty} \frac{p^{w}}{\left(p+R_{e x c}-d_{k}\right)^{2 n+1}\left(p+R_{e x c}+d_{k}\right)^{a-1}} d p\right. \\
& =2 m_{1} \lambda P_{I} \sum_{n=0}^{\frac{a}{2}-1}\left(\begin{array}{c}
\frac{a}{2}-1 \\
n
\end{array}\right) B\left(R_{e x c}+d_{k}\right)^{1-a} \\
& \times\left(R_{e x c}-d_{k}\right)^{w-2 n} B(w+1, a+2 n-w-1)_{2} F_{1}\left(a-1, w+1, a+2 n, \frac{2 d_{k}}{R_{e x c}+d_{k}}\right)
\end{aligned}
$$

which follows from [38, 3.197.1]. In the same way, $E_{I}\left\{I_{3_{k}}\right\}$ is given by (51) by replacing $m_{1}$ with $m_{2}$.

2) $a \neq 2 m$, where $m=2,3,4, \ldots$ In this case, the exponent $\frac{a}{2}-1$ of the numerator of the second integral in (49) is fractional and so we cannot rely on the known binomial expansion formula that works only for integer exponents. Instead, we can rely on Newton's generalized binomial expansion formula for fractional exponents. In particular, if $x$ and $y$ are real numbers with $|x|>|y|$, and $r$ is a complex number, it holds that [38]

$$
(x+y)^{r}=\sum_{n=0}^{\infty} \frac{(r)_{n}}{n !} x^{r-n} y^{n} .
$$

Now, we see that the variable $u$ of the integral $\int_{0}^{\frac{1}{\left(l_{1}-d_{k}\right)^{2}}-\frac{1}{\left(l_{1}+d_{k}\right)^{2}}} \frac{\left(u+\frac{1}{\left(l_{1}+d_{k}\right)^{2}}\right)^{\frac{a}{2}-1}}{\sqrt{u} \sqrt{\frac{1}{\left(l_{1}-d_{k}\right)^{2}}-\frac{1}{\left(l_{1}+d_{k}\right)^{2}}}} d u$ of (49) takes values in the interval $\left[0, \frac{1}{\left(l_{1}-d_{k}\right)^{2}}-\frac{1}{\left(l_{1}+d_{k}\right)^{2}}\right]$. Due to this, we distinguish two subcases:

i) The maximum value that $u$ takes, which is $\frac{1}{\left(l_{1}-d_{k}\right)^{2}}-\frac{1}{\left(l_{1}+d_{k}\right)^{2}}$, is smaller than the term $\frac{1}{\left(l_{1}+d_{k}\right)^{2}}$ of the 
enumerator of $\int_{0}^{\frac{1}{\left(l_{1}-d_{k}\right)^{2}}-\frac{1}{\left(l_{1}+d_{k}\right)^{2}}} \frac{\left(u+\frac{1}{\left(l_{1}+d_{k}\right)^{2}}\right)^{\frac{a}{2}-1}}{\sqrt{u} \sqrt{\frac{1}{\left(l_{1}-d_{k}\right)^{2}}-\frac{1}{\left(l_{1}+d_{k}\right)^{2}}-u}} d u$, which means that

$$
\frac{1}{\left(l_{1}-d_{k}\right)^{2}}-\frac{1}{\left(l_{1}+d_{k}\right)^{2}}<\frac{1}{\left(l_{1}+d_{k}\right)^{2}} \Rightarrow l_{1}^{2}-6 l_{1} d_{k}+d_{k}^{2}>0
$$

The two roots of the second degree polynomial of (53) are

$$
l_{1_{1,2}}=(3 \pm 2 \sqrt{2}) d_{k}
$$

Hence,

$$
l_{1}^{2}-6 l_{1} d_{k}+d_{k}^{2}>0 \Rightarrow l_{1}<(3-2 \sqrt{2}) d_{k} \quad \text { or } \quad l_{1}>(3+2 \sqrt{2}) d_{k}
$$

Due to the fact that $l_{1}$ takes values in the interval $\left[R_{e x c}, \infty\right)$, according to the first integral of (49), only the right-side root of (55) satisfies the condition. Hence,

$$
\frac{1}{\left(l_{1}-d_{k}\right)^{2}}-\frac{1}{\left(l_{1}+d_{k}\right)^{2}}<\frac{1}{\left(l_{1}+d_{k}\right)^{2}} \Rightarrow l_{1}^{2}-6 l_{1} d_{k}+d_{k}^{2}>0 \stackrel{l_{1} \in\left[R_{\text {exc }}, \infty\right)}{\Rightarrow} R_{\text {exc }}>(3+2 \sqrt{2}) d_{k} .
$$

When (56) holds, the numerator of $\int_{0}^{\frac{1}{\left(l_{1}-d_{k}\right)^{2}}-\frac{1}{\left(l_{1}+d_{k}\right)^{2}}} \frac{\left(u+\frac{1}{\left(l_{1}+d_{k}\right)^{2}}\right)^{\frac{a}{2}-1}}{\sqrt{u} \sqrt{\frac{1}{\left(l_{1}-d_{k}\right)^{2}}-\frac{1}{\left(l_{1}+d_{k}\right)^{2}}-u}} d u$ can be expanded according to (52), which means that

$$
\begin{aligned}
& \int_{0}^{\frac{1}{\left(l_{1}-d_{k}\right)^{2}}-\frac{1}{\left(l_{1}+d_{k}\right)^{2}}} \frac{\left(u+\frac{1}{\left(l_{1}+d_{k}\right)^{2}}\right)^{\frac{a}{2}-1}}{\sqrt{u} \sqrt{\frac{1}{\left(l_{1}-d_{k}\right)^{2}}-\frac{1}{\left(l_{1}+d_{k}\right)^{2}}-u}} d u \\
& =\sum_{n=0}^{\infty} \frac{\left(\frac{a}{2}-1\right)_{n}}{n !}\left[\frac{1}{\left(l_{1}+d_{k}\right)^{2}}\right]^{\frac{a}{2}-1-n} \int_{0}^{\frac{1}{\left(l_{1}-d_{k}\right)^{2}}-\frac{1}{\left(l_{1}+d_{k}\right)^{2}}} \frac{u^{n-\frac{1}{2}}}{\sqrt{\frac{1}{\left(l_{1}-d_{k}\right)^{2}}-\frac{1}{\left(l_{1}+d_{k}\right)^{2}}-u}} d u \\
& =\sum_{n=0}^{\infty} \frac{\left(\frac{a}{2}-1\right)_{n}}{n !}\left[\frac{1}{\left(l_{1}+d_{k}\right)^{2}}\right]^{\frac{a}{2}-1-n}\left[\frac{4 l_{1} d_{k}}{\left(l_{1}-d_{k}\right)^{2}\left(l_{1}+d_{k}\right)^{2}}\right]^{n} B\left(\frac{1}{2}, n+\frac{1}{2}\right),
\end{aligned}
$$

which follows from [38, 3.191.1]. By plugging (57) into (49) and following the same steps as in (51), we get

$$
\begin{aligned}
E_{I}\left\{I_{1_{k}}\right\} & =2 m_{1} \lambda P_{I} \sum_{n=0}^{\infty} \frac{\left(\frac{a}{2}-1\right)_{n}}{n !} B\left(\frac{1}{2}, n+\frac{1}{2}\right)\left(4 d_{k}\right)^{n} \sum_{w=0}^{n+1}\left(\begin{array}{c}
n+1 \\
w
\end{array}\right) R_{e x c}^{n+1-w}\left(R_{e x c}+d_{k}\right)^{1-a} \\
& \times\left(R_{e x c}-d_{k}\right)^{w-2 n} B(w+1, a+2 n-w-1)_{2} F_{1}\left(a-1, w+1, a+2 n, \frac{2 d_{k}}{R_{e x c}+d_{k}}\right) .
\end{aligned}
$$

Obviously, (58) is not in closed form since the first series grows to infinity. However, we can truncate it 
and take a finite number of terms to approximate the series. In Section IV, we numerically show that a number of terms as low as 15 is adequate so that the examined performance metrics closely match the Monte Carlo simulations in the high-SINR region.

ii) If $R_{\text {exc }} \leqslant(3+2 \sqrt{2}) d_{k}$, it holds that there is a range of values of $l_{1}$ for which the maximum value $\frac{1}{\left(l_{1}-d_{k}\right)^{2}}-\frac{1}{\left(l_{1}+d_{k}\right)^{2}}$ of $u$ is smaller, greater, or equal to the term $\frac{1}{\left(l_{1}+d_{k}\right)^{2}}$ of the enumerator of $\int_{0}^{\frac{1}{\left(l_{1}-d_{k}\right)^{2}}-\frac{1}{\left(l_{1}+d_{k}\right)^{2}}} \frac{\left(u+\frac{1}{\left(l_{1}+d_{k}\right)^{2}}\right)^{\frac{a}{2}-1}}{\sqrt{u} \sqrt{\frac{1}{\left(l_{1}-d_{k}\right)^{2}}-\frac{1}{\left(l_{1}+d_{k}\right)^{2}}-u}} d u$, based on (54) and since $l_{1} \in\left[R_{e x c}, \infty\right)$. Due to the analytical difficulty of this case, we consider the following. We take a look at Fig. 1 and we observe that source $k$ is under the effect of asymmetric interference since it is not located at the center of the circle with radius $R_{e x c}$. In particular, due to this asymmetry there are two values of the smallest possible distance between source $k$ and any interferer that are diametrically opposite. The first distance (smallest one), as we observe from Fig. 1, is equal to $R_{e x c}-d_{k}$ and the second one is equal to $R_{e x c}+d_{k}$. Hence, we can consider two bounds of the interference that affects source $k$. The first bound corresponds to the assumption that source $k$ is located at the center of a circular exclusion region with radius $R_{e x c}-d_{k}$ and the second bound corresponds to the assumption that source $k$ is located at the center of a circular exclusion region with radius $R_{e x c}+d_{k}$. The first bound, which corresponds to the radius $R_{e x c}-d_{k}$, gives a higher value than the actual interference that source $k$ experiences, whereas the second bound, which corresponds to the radius $R_{e x c}+d_{k}$, gives a smaller value than the actual one. Under these considerations, we can name these two bounds of the interference as Higher Value (HV), which corresponds to the exclusion region with radius $R_{e x c}-d_{k}$, and Lower Value (LV), which corresponds to the exclusion region with radius $R_{e x c}+d_{k}$. This means that [41, Eq. (8)] (according to Campbell's theorem)

$$
E_{I}\left\{I_{1_{k}}\right\}_{H V}=\frac{2 \pi m_{1} \lambda P_{I}}{a-2}\left(R_{e x c}-d_{k}\right)^{2-a} \quad \text { and } \quad E_{I}\left\{I_{1_{k}}\right\}_{L V}=\frac{2 \pi m_{1} \lambda P_{I}}{a-2}\left(R_{e x c}+d_{k}\right)^{2-a}
$$

Now, we can approximate $E_{I}\left\{I_{1_{k}}\right\}$ by taking the average value of $E_{I}\left\{I_{1_{k}}\right\}_{H V}$ and $E_{I}\left\{I_{1_{k}}\right\}_{L V}$. Hence,

$$
E_{I}\left\{I_{1_{k}}\right\} \approx \frac{1}{2}\left(E_{I}\left\{I_{1_{k}}\right\}_{H V}+E_{I}\left\{I_{1_{k}}\right\}_{L V}\right) \stackrel{(c)}{=} \frac{\pi \lambda m_{1} P_{I}}{a-2}\left[\left(R_{e x c}-d_{k}\right)^{2-a}+\left(R_{e x c}+d_{k}\right)^{2-a}\right]
$$

where in $(c)$ we use (59). The appropriateness of (60) as an approximation of the actual average interference is verified against Monte Carlo simulations in Section IV for the examined performance metrics. Furthermore, we again note that $E_{I}\left\{I_{3_{k}}\right\}$ is given by the expression of $E_{I}\left\{I_{1_{k}}\right\}$ by replacing $m_{1}$ with $m_{2}$, which concludes the proof of Proposition 3. 


\section{REFERENCES}

[1] S. Catti, S. Gollacota, and D. Katabi, "Embracing wireless interference: Analog network coding," ACM SIGCOMM, 2007.

[2] Q. Zhou, et. al., "Decode-and-Forward Two-Way Relaying with Network Coding and Opportunistic Relay Selection," IEEE Trans. Commun., vol. 58, no. 11, pp. 3070-3076, June 2010.

[3] T. Akino, P. Popovsi, and V. Tarokh, "Optimized constellations for two-way wireless relaying with physical network coding," IEEE Journal Sel. Areas. On Commun., vol. 27, no. 5, pp. 773-787, June. 2009.

[4] S. Shukla, V. Muralidharan, and S. Rajan, "Wireless Network-Coded Accumulate-Compute and Forward Two-Way Relaying," IEEE Trans. Veh. Technol., accepted for publication.

[5] Y. Yang, H. Honglin, X. Jing, and M. Guoqiang, "Relay technologies for WiMax and LTE-advanced mobile systems," IEEE Commun. Magazine, vol. 47, no. 10, pp. 100-105, Oct. 2009.

[6] R. Vaze and R. W. Heath, "On the Capacity and Diversity-Multiplexing Tradeoff of the Two-Way Relay Channel," IEEE Trans. Inf. Theory, vol. 57, no. 7, pp. 4219-4234, July 2011.

[7] X. Cheng et. al., "Two-Way Full-Duplex Amplify-and-Forward Relaying,” MILCOM, San Diego, United States, 2013.

[8] R. Wang and M. Tao, "Joint Source and Relay Precoding Designs for MIMO Two-Way Relaying Based on MSE Criterion," IEEE Trans. Signal Process., vol. 60, no. 3, pp. 1352-1365, Dec. 2011.

[9] M. Tao and R. Wang, "Linear Precoding for Multi-Pair Two-Way MIMO Relay Systems With Max-Min Fairness," IEEE Trans. Signal Process., vol. 60, no. 10, pp. 5361-5370, Sep. 2012.

[10] M. Zhou, Q. Cui, R. Jantti, and X. Tao, "Energy-Efficient Relay Selection and Power Allocation for Two-Way Relay Channel with Analog Network Coding," IEEE Comm. Letters, vol. 16, no. 6, pp. 816-819, June 2012.

[11] X. Ji, B. Zheng, Y. Cai, and L. Zou, "On the Study of Half-Duplex Asymmetric Two-Way Relay Transmission Using an Amplify-andForward Relay," IEEE Comm. Letters, vol. 61, no. 4, pp. 1649-1664, May 2012.

[12] L. Song,, Y. Li, A. Huang, B. Jiao, and A. V. Vasilakos, "Differential modulation for bidirectional relaying with analog network coding," IEEE Trans. Signal Processing, vol. 58, no. 7, pp. 3933-3938, July 2010.

[13] G. Kaddoum and F. Shokraneh (2015), "Analog Network Coding for Multi-User Multi-Carrier Differential Chaos Shift Keying Communication System,” IEEE Trans. Wirel. Commun., vol. 14, no. 3, pp. 1492-1505, March 2015.

[14] M. J. Siavoshani, S. Mohajer, C. Fragouli, and S. N. Diggavi, "On the Capacity of Noncoherent Network Coding," IEEE Trans. Inf. Theory, vol. 57, no. 2, pp. 1046-1066, Feb. 2011.

[15] W. Guan and K. R. Liu, "On analysis of wireless uplink using analog network coding with non-coherent modulations," Proceedings of IEEE GLOBECOM, 2012.

[16] C. Wang, T. C.-K. Liu. and X. Dong, "Impact of Channel Estimation Error on the Performance of Amplify-and-Forward Two-Way Relaying," IEEE Trans. Veh. Technol., vol. 61, no. 3, pp. 1197-2007, March 2012.

[17] A. Goldsmith, Wireless Communications, Cambridge University Press, Aug. 2005.

[18] F.S. Tabataba, P. Sadeghi, C. Hucher, and M.R. Pakravan, "Impact of Channel Estimation Errors and Power Allocation on Analog Network Coding and Routing in Two-Way Relaying," IEEE Trans. Veh. Technol., vol. 61, no. 7, pp. 3223-3239, Sep. 2012.

[19] S. S. Ikki and S. Aïssa, "Two-Way Amplify-and-Forward Relaying with Gaussian Imperfect Channel Estimations," IEEE Commun. Letters, vol. 16, no. 7, pp. 956-959, July 2012.

[20] X. Liang, S. Jin, W. Wang, X. Gao, and K.-K. Wong, "Outage probability of amplify-and-forward two-way relay interference-limited systems," IEEE Trans. Veh. Technol., vol. 61, no. 6, pp. 2221-2231, June 2013. 
[21] J. Shen, N. Sha, Y. Cai, C. Cai, and W. Yang, "Outage probability of two-way amplify-and-forward relaying system with interferencelimited relay," Wireless Commun. Signal Process., 2011.

[22] S. S. Ikki and S. Aissa, "Performance analysis of two-way amplify-and- forward relaying in the presence of co-channel interference," IEEE Trans. Commun., vol. 60, no. 4, pp. 933-939, Apr. 2012.

[23] L. Yang, K. Qarage, E. Serpedin, and M.-S. Alouini, "Performance Analysis of Amplify-and-Forward Two-Way Relaying with CoChannel Interference and Channel Estimation Error," IEEE Trans. Commun., vol. 61, no. 6, pp. 2221-2231, June 2013.

[24] A. Ghosh, N. Mangalvedhe, R. Ratasuk, B. Mondal, M. Cudak, E. Visotsky, T. A. Thomas, J. G. Andrews, P. Xia, H. S. Jo, H. S. Dhillon, and T. D. Novlan, "Heterogeneous cellular networks: From theory to practice," IEEE Commun. Mag., vol. 50, no. 6, pp. 54-64, July 2012.

[25] J. Andrews, "Seven ways that HetNets are a cellular paradigm shift," IEEE Commun. Mag., vol. 51, no. 3, pp. 136-144, Mar. 2013.

[26] M. Z. Win, P. C. Pinto, and L. A. Shepp, “A mathematical theory of network interference and its applications," Proc. of the IEEE, vol. 97, no. 2, pp. 205-230, Feb. 2009.

[27] P. C. Pinto and M. Z. Win, "Communication in a Poisson field of interferers - Part I: Interference distribution and error probability," IEEE Trans. Wireless Commun., vol. 9, no. 7, pp. 2176-2186, July 2010.

[28] R. Heath, M. Kountouris, and T. Bai,"Modeling heterogeneous network interference using Poisson Point Processes,” IEEE Trans. Signal Process., vol. 61, no. 16, pp. 4114-4126, Aug. 2013.

[29] M. Kountouris and Pappas, "Approximating the Interference Distribution in Large Wireless Networks," ISWCS, Barcelona, Spain, 2014.

[30] M. A. Mohammadi, H. A. Suraweera, and X. Zhou, "Outage probability of wireless ad hoc networks with cooperative relaying," IEEE Global Commun. Conf., pp. 4632-4638, Dec. 2012.

[31] A. Guidotti, V. Buccigrossi, M. Di Renzo, G. E. Corazza, and F. Santucci, "Outage and symbol error probabilities of dual-hop AF relaying in a Poisson field of interferers," IEEE Wireless Commun. Netw. Conf., pp. 3704-3709, Apr. 2013.

[32] R. Tanbourgi, H. Jakel, and F. K. Jondral, "Cooperative relaying in a Poisson field of interferers: A diversity order analysis," IEEE Int. Symp. Inform. Theory, pp. 3100-3104, July 2013.

[33] V. A. Aalo, K. P. Peppas, G. Efthymoglou, M. Alwakeel, and S. Alwakeel, "Serial amplify-and-forward relay transmission systems in Nakagami-m fading channels with a Poisson interference field,” IEEE Trans. Veh. Technol., vol. 63, no. 5, pp. $2183-2196$, June 2014.

[34] M. Di Renzo and W. Lu, "End-to-end error probability and diversity analysis of AF-based dual-hop cooperative relaying in a Poisson field of interferers at the destination," IEEE Trans. Wireless Commun., vol. 14, no. 1, pp. 15-32, Jan. 2015.

[35] M. Di Renzo and W. Lu, "On the Diversity Order of Selection Combining Dual-Branch Dual-Hop AF Relaying in a Poisson Field of Interferers at the Destination,” IEEE Trans. Veh. Technol., vol. 64, no. 4, pp. 1620-1628, April 2015.

[36] H. Inatelkin, C. Mung., H. V. Poor, and S. B. Wicker, "On unbounded path-loss models: effects of singularity on wireless network performance," IEEE Journal on Sel. Areas of Commun., vol. 27, no. 7, pp. 1078-1092, Sep. 2009.

[37] M. Haenggi, Stochastic Geometry for Wireless Networks, Cambridge University Press, Nov. 2012.

[38] I. S. Gradshteyn and I. M. Ryzhik, Table of Integrals, Series, and Products, 7th edition. Academic, 2007.

[39] M. Abramovitz and I. A. Stegun, Handbook of Mathematical Functions with Formulas, Graphs, and Mathematical Tables, 9th edition. Dover, 1972.

[40] Z. Wang and G.B. Giannakis, "A simple and general parameterization quantifying performance in fading channels," IEEE Trans. Wireless Commun., vol. 51, no. 8, pp. 1389-1398, Aug. 2003.

[41] H.S. Lichte, S. Valentin, and H. Karl, "Expected interference in wireless networks with geometric path loss: a closed-form approximation," IEEE Commun. Letters, vol. 14, no. 2, pp. 130-132, Feb. 2010.

[42] J. Proakis, Digital Communications, 4th ed. New York: McGraw-Hill, August 2000. 
[43] R. H.Y. Louie, L. Yonghui, and B. Vucetic, "Practical physical layer network coding for two-way relay channels: performance analysis and comparison," IEEE Trans. Wireless Commun., vol. 9, no. 2, pp. 764-777, Feb. 2010. 